March 8, 2015

Yellowstone Convention Center - Big Sky, MT

\title{
Large Volume, Optical and Opto- Mechanical Metrology Techniques for ISIM on JWST
}

Theo Hadjimichael

Optics Branch

NASA Goddard Space Flight Center

Greenbelt, Maryland 


\section{Outline}

I. INTRODUCTION

II. PURPOSE AND ALIGNMENT PLAN III. REQUIREMENTS

IV. METHODS AND TOOLS

V. TESTS, MEASUREMENT AND SETUP

VI. ANALYSIS

VII.RESULTS

VIII.DATABASES

IX. CONCLUSION 
ISIM consists of:

- Five sensor systems

- MIRI, NIRCam, NIRSpec, NIRISS, FGS

- Nine instrument support systems:

- Optical metering structure system

- Electrical Harness System

- Harness Radiator System

- ISIM Electronics Compartment (IEC)

- Cryogenic Thermal Control System

- Command and Data Handling System $(\mathrm{ICDH})$

- ISIM Remote Services Unit (IRSU)

- Flight Software System

- Operations Scripts System 


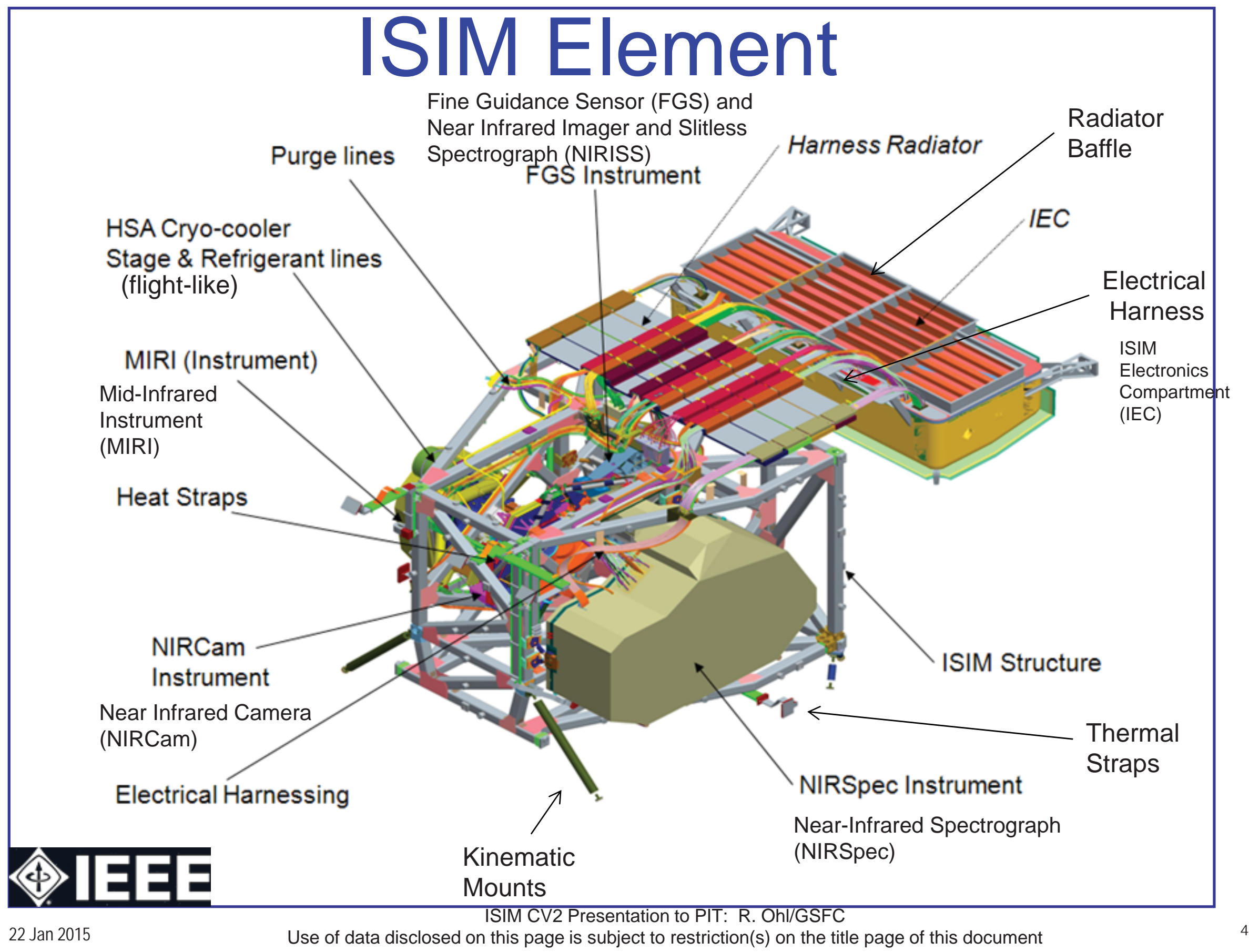




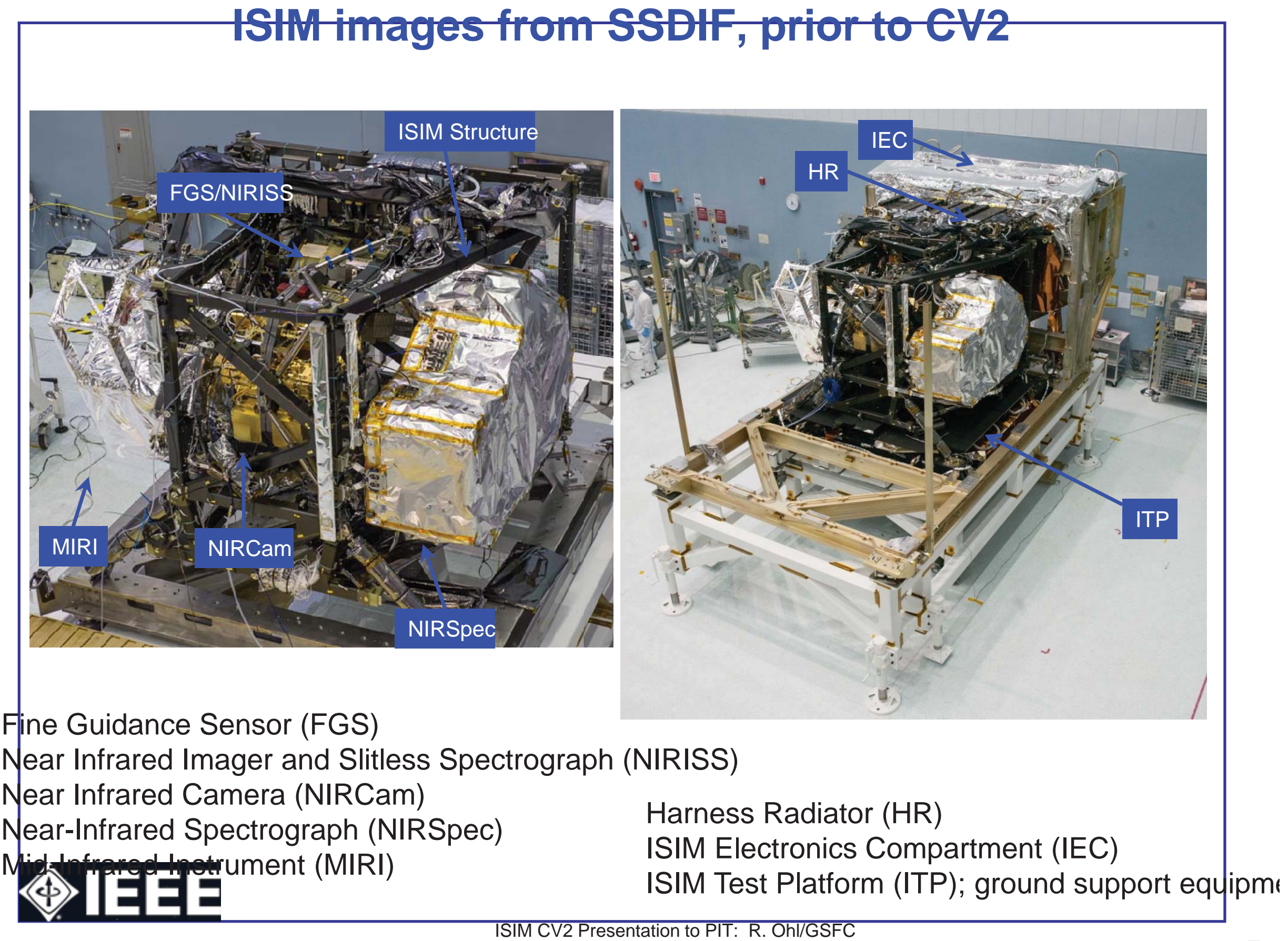




\section{ASMIF and ISIM}

- Instrument were built on a GSE (Ambient Science Insturment Mechanical Interface Fixture, ASMIF) that mimics the ISIM-SI interface

- Identical Science Instrument Interface Plates (SIIP) were fabricated for the ASMIFs and ISIM structure
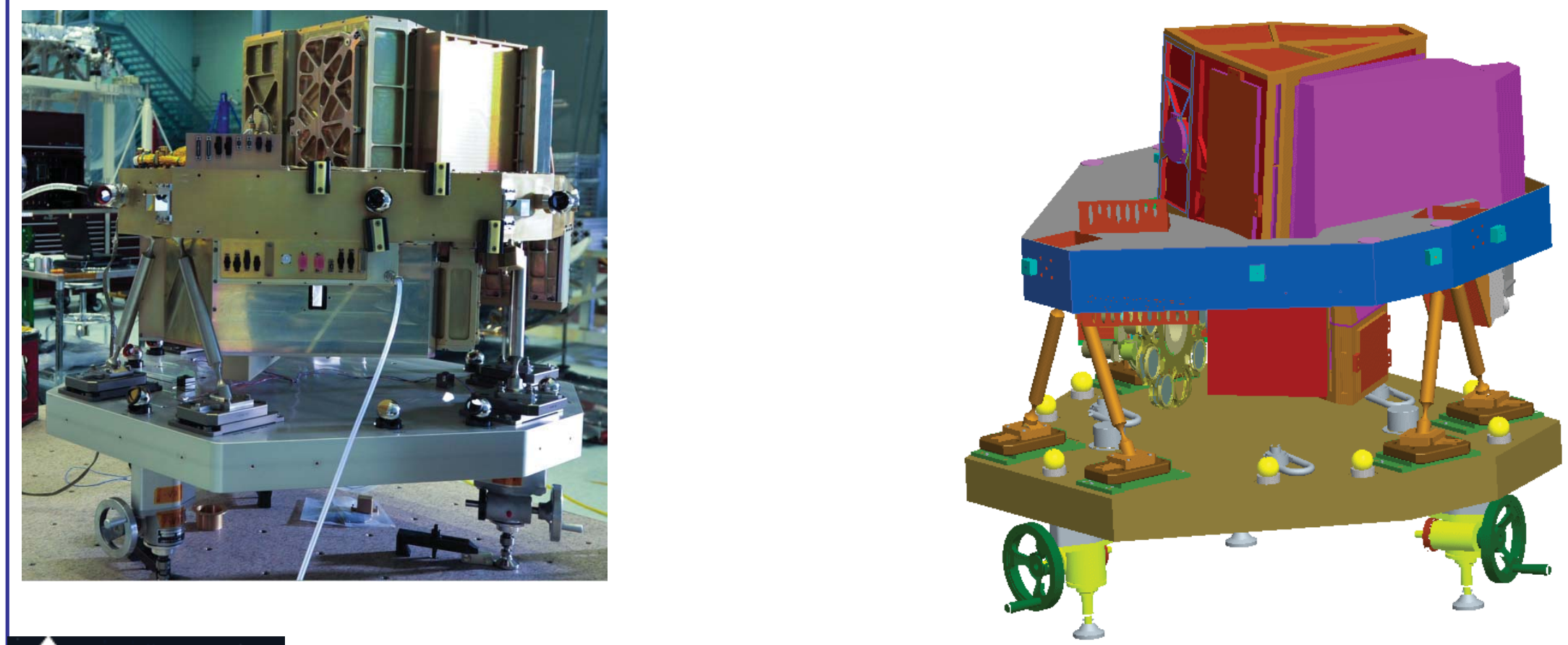


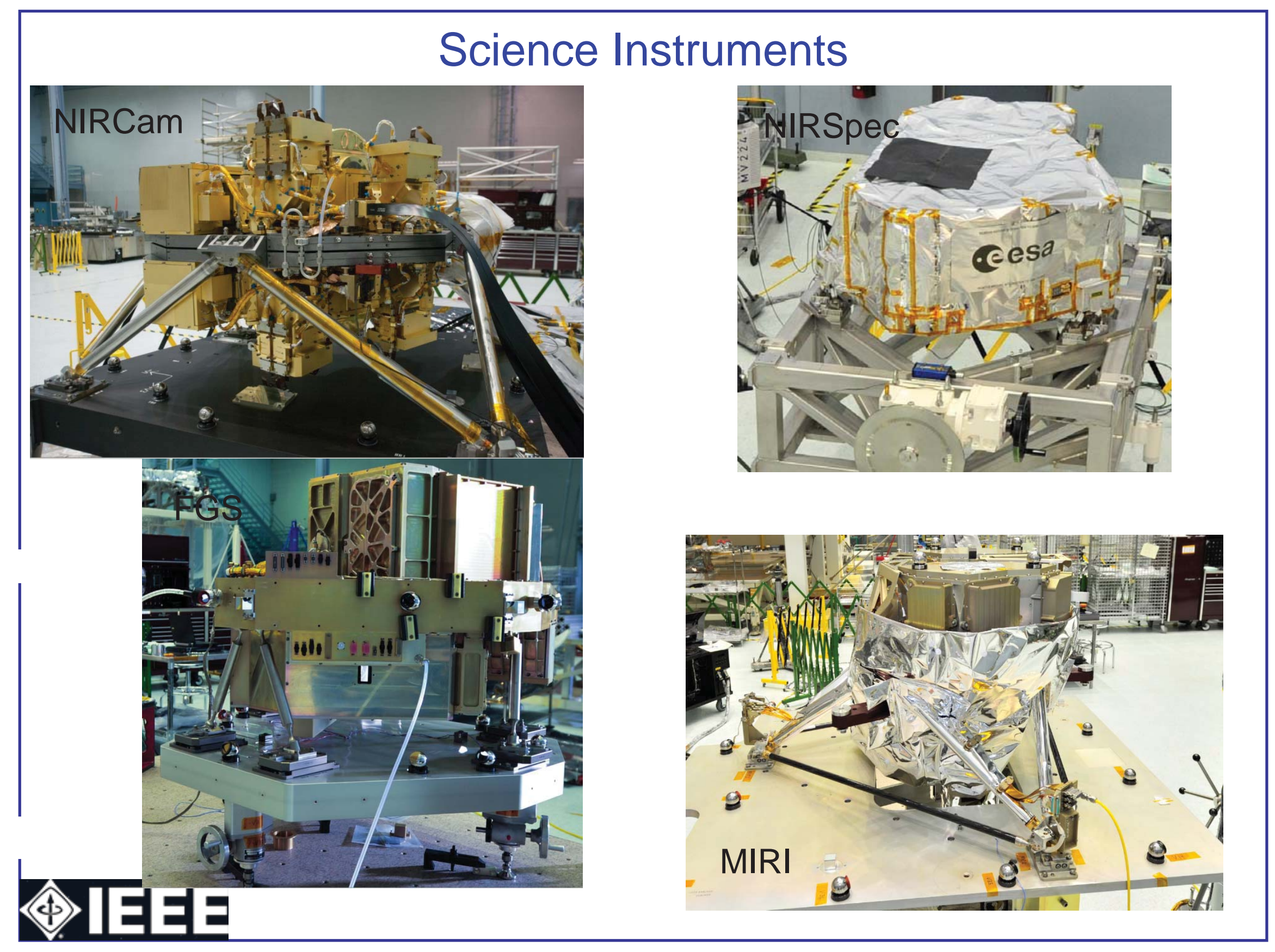




\section{Ground Support Equipment (GSE)}

Master and ISIM alignment target fixture (MATF/IATF) Targets tracked and used to align optical simulator to ISIM

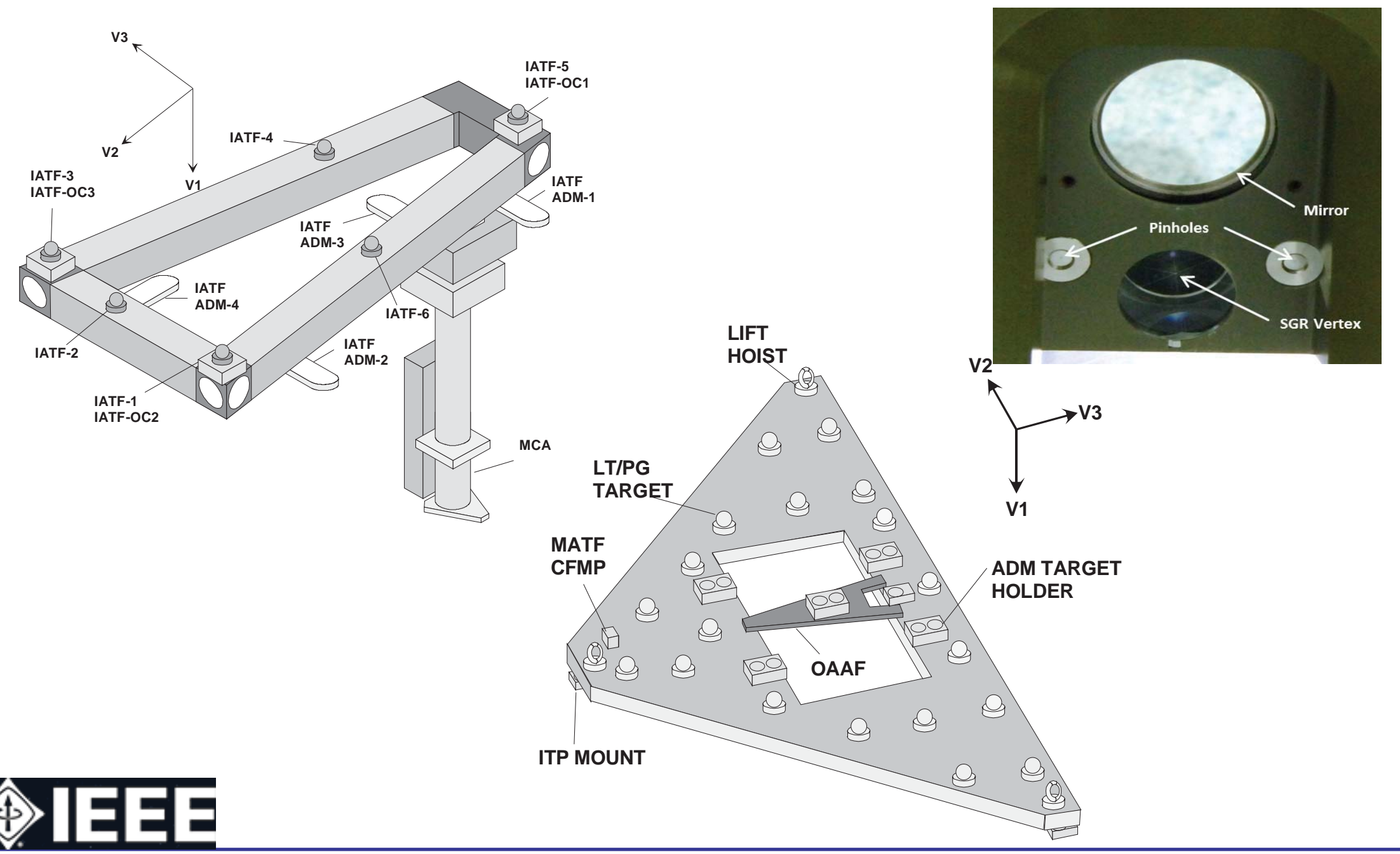




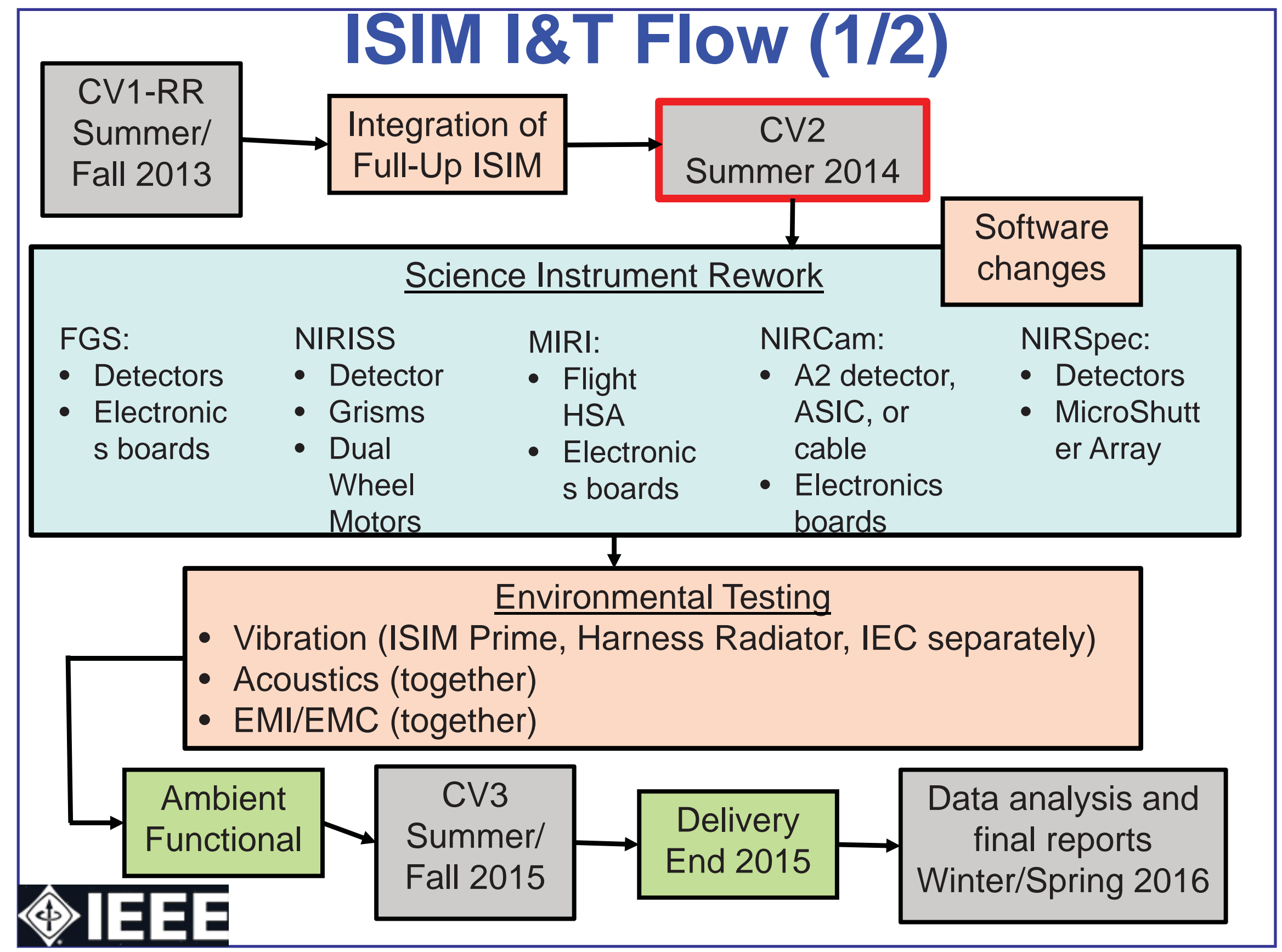




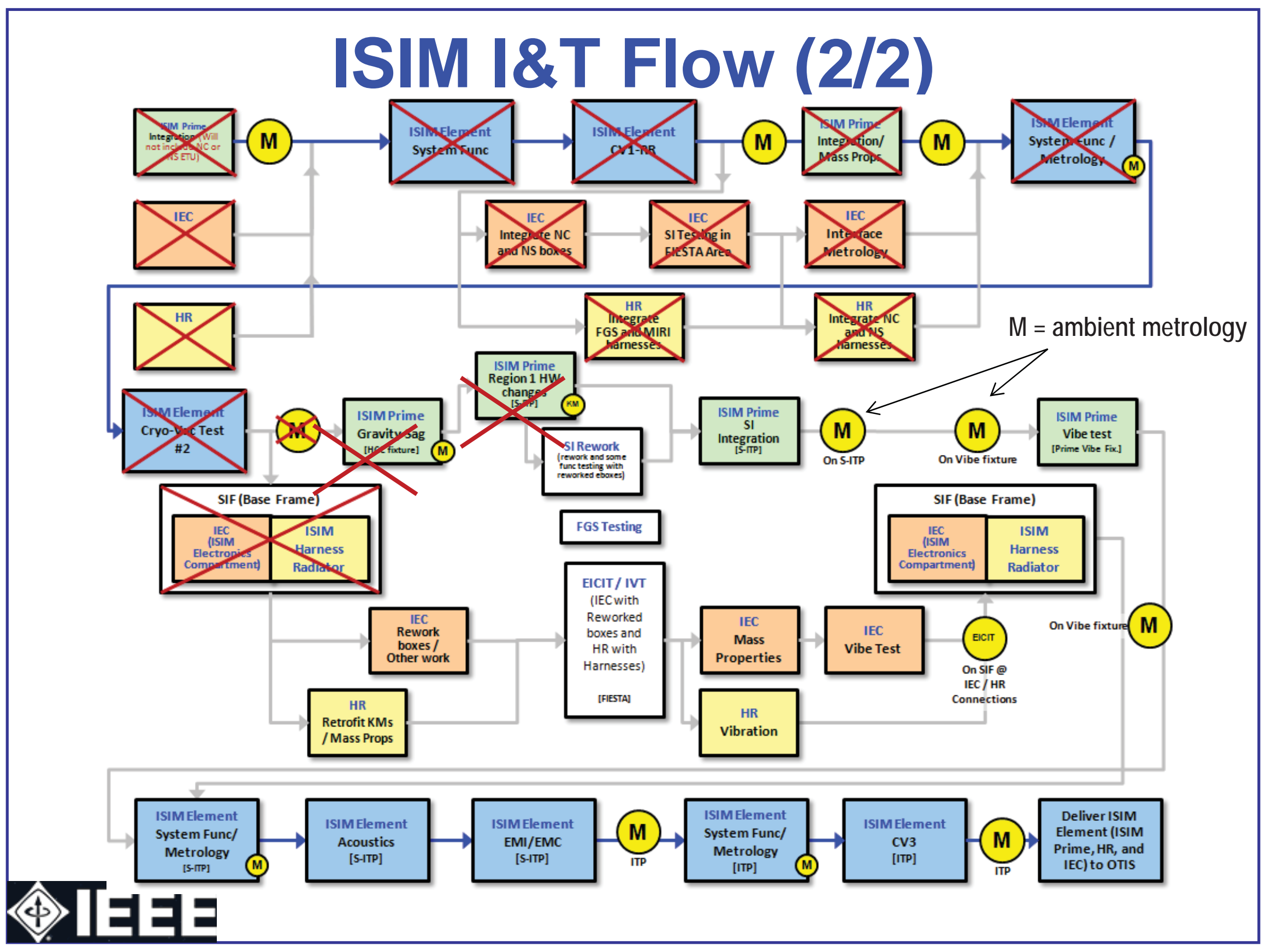

22 Jan 2015 


\section{Purpose of Work}

- Verify the ambient 6 degree of freedom (DOF) alignment of the SIs to the ISIM structure

- This is done using various metrology targets located on the ISIM structure and each SI

- Interchangable spherically Mounted Retroreflector (SMR) and Tooling Ball (TB) nests

- Optical alignment cubes

- Locations calibrated relative to precision mechanical interfaces

- In addition, each instrument contains a pupil alignment reference (PAR) that is measured near the nominal predicted ambient OTE exit pupil location

- Build a comprehensive database of tracking targets through all environmental testing 


\section{Requirements}

- Uncertainties are determined via a bottoms up estimation and are compared to expectations allocated from the top-level ISIM requirements

- Overall test requirement is that the measured SI nests and cube locations are at their predicted locations within the 2-sigma uncertainties of:

- The SI optical bench (OB) and Ground support equipment (GSE) measured locations when integrated to ISIM

- The SI OB measured locations while integrated to the ASMIF structure

- Finite element modeling (FEM) of the above two orientations with respect to gravity

- Misalignment associated with small differences in ASMIF and Structure precision mount interfaces 


\section{Methods and Tools}

- A variety of measurement tools are used depending on the application and requirements

- Tools used include: Leica laser trackers (LT), Nikon laser radar (LR), Leica Wild T3000 theodolites and a Koll Morgen alignment telescope (AT)

- LR is typically used for measurements of the SI optical benches (OB) and ISIM structure TB targets

- LT was used for PAR measurements primarily for its ability to track an SMR for alignment purposes

- Theodolites were used for all optical cube face measurements

- Photogrammetry Cameras used during both ambient and cryogenic testing 


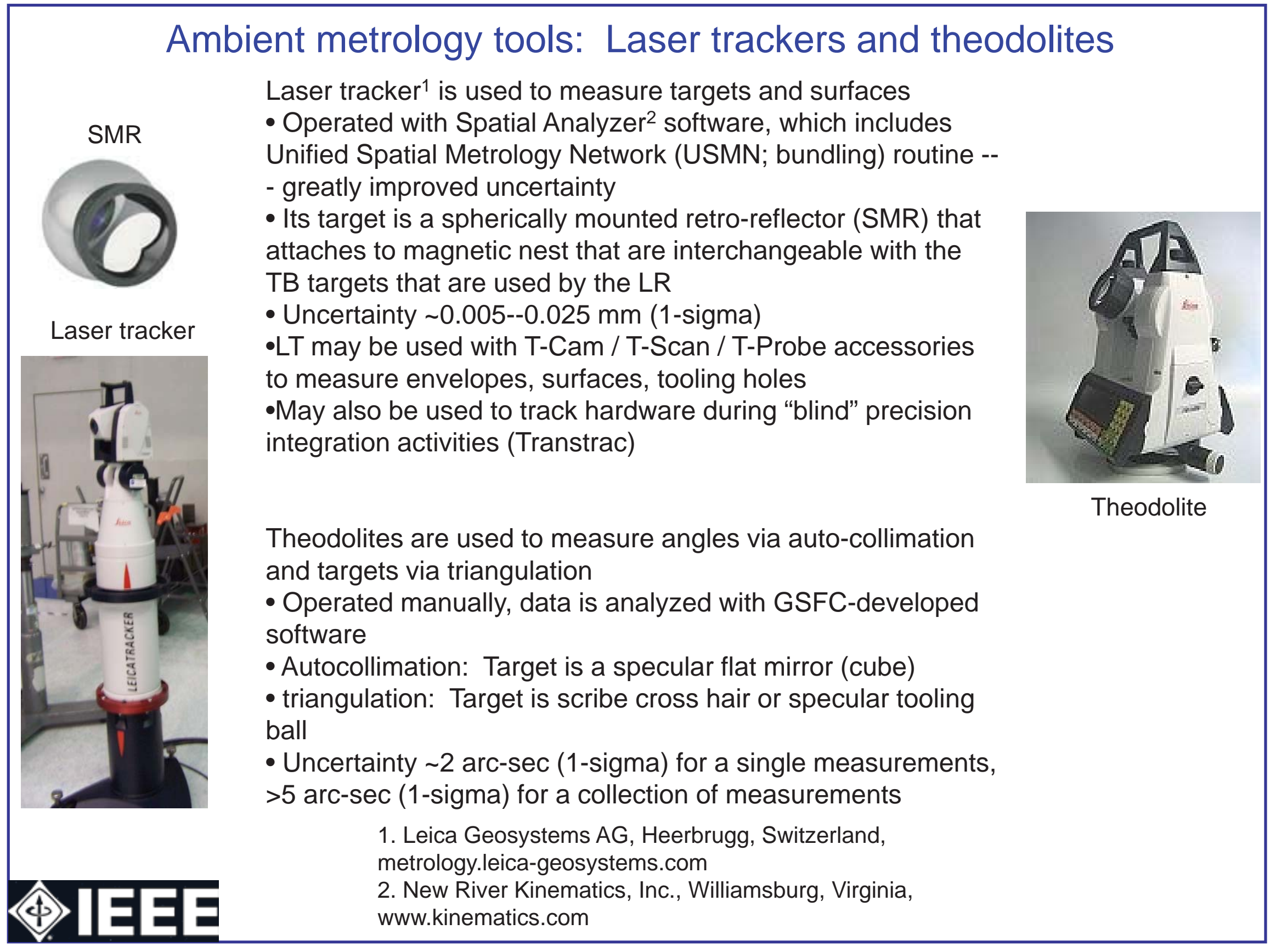


Slide 14

pc1 add LR photo description

pcoulter, 7/8/2014 


\section{Ambient metrology tool: "Laser radar"}

Laser $\operatorname{radar}^{1}$ (LIDAR) is used to measure targets and surfaces

- $\quad$ Operated with Spatial Analyzer software

- Its target is diffuse surface (mechanical surface; matt finish), reflective tooling ball, specular mirror, or high-quality tooling hole

- Uncertainty $\sim 0.010 \mathrm{~mm}$ in range (1-sigma), $\sim 0.015 \mathrm{~mm}$ per meter in azimuth and elevation (1-sigma)

- $\quad$ Laser Radar scans much faster than Laser Tracker with T-Probe

- USMN-compatible

- At ambient, used for:

- Used for prescription and alignment measurement for large optics (radius, aperture, etc.)

- Envelope scans

- Tooling ball targets on large assemblies

1. Nikon Metrology Inc, http://www.nikonmetrology.com/en_US

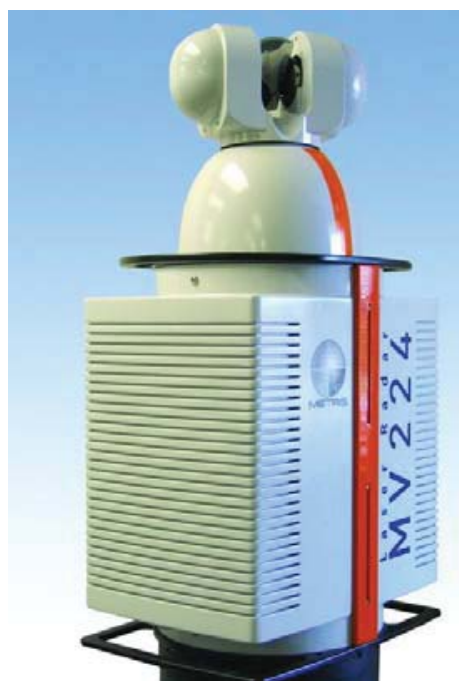

Laser radar (LIDAR)
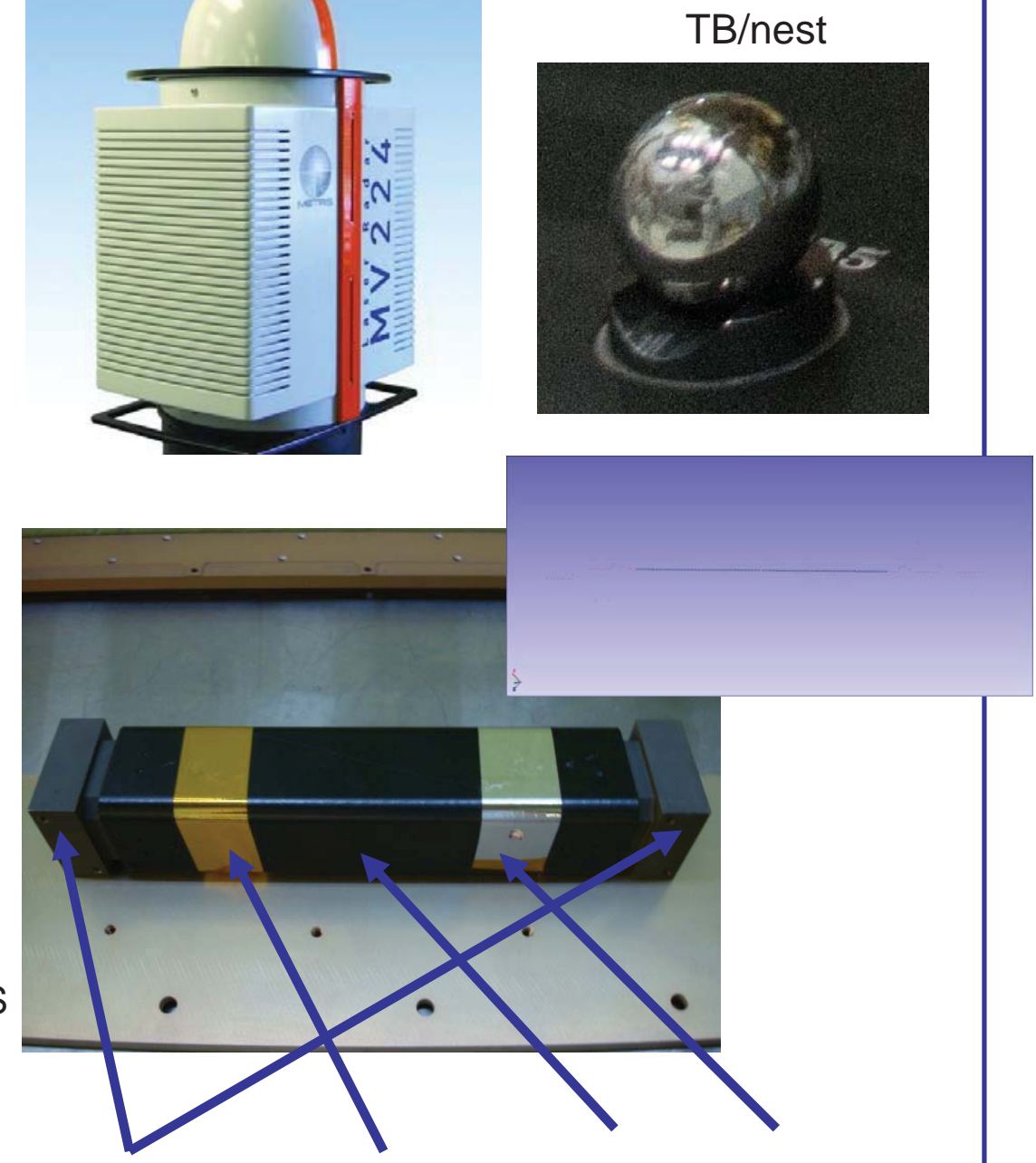


\section{Photogrammetry Overview}

- 3 dimensional metrology system

- Uses triangulation to locate custom targets in 3 coordinates

- Requires multiple camera locations

- Solves for the camera locations and coordinates of the targets simultaneously through the bundling procedure contained in
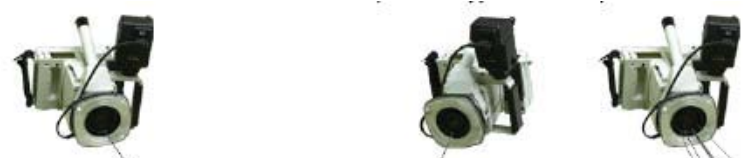
the V-STARS software, proprietary software owned by Geodetic Systems Inc.

- Software contains calibration algorithms to calibrate internal camera errors

- Geometrically diverse scalebars provide scale

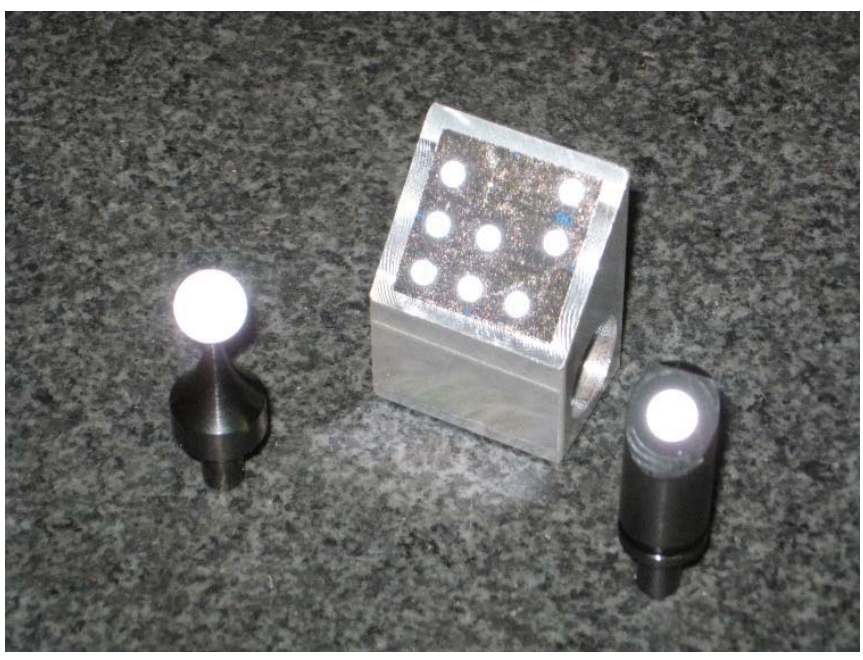




\section{Measurement Setups}

- Measurements conducted in the NASA GSFC Space Systems Development and Integration Facility (SSDIF) and Space Environment Simulator (SES) chamber

- Tests

- GSE only ambient/cryogenic characterization

- Full multi-station LR measurements of SI OB and ISIM structure targets

- Full suite of theodolite measurements of all SI OB cubes and ISIM structure cubes

- Five sets of measurements taken at each station

- Resulting final values used for PAR measurements 


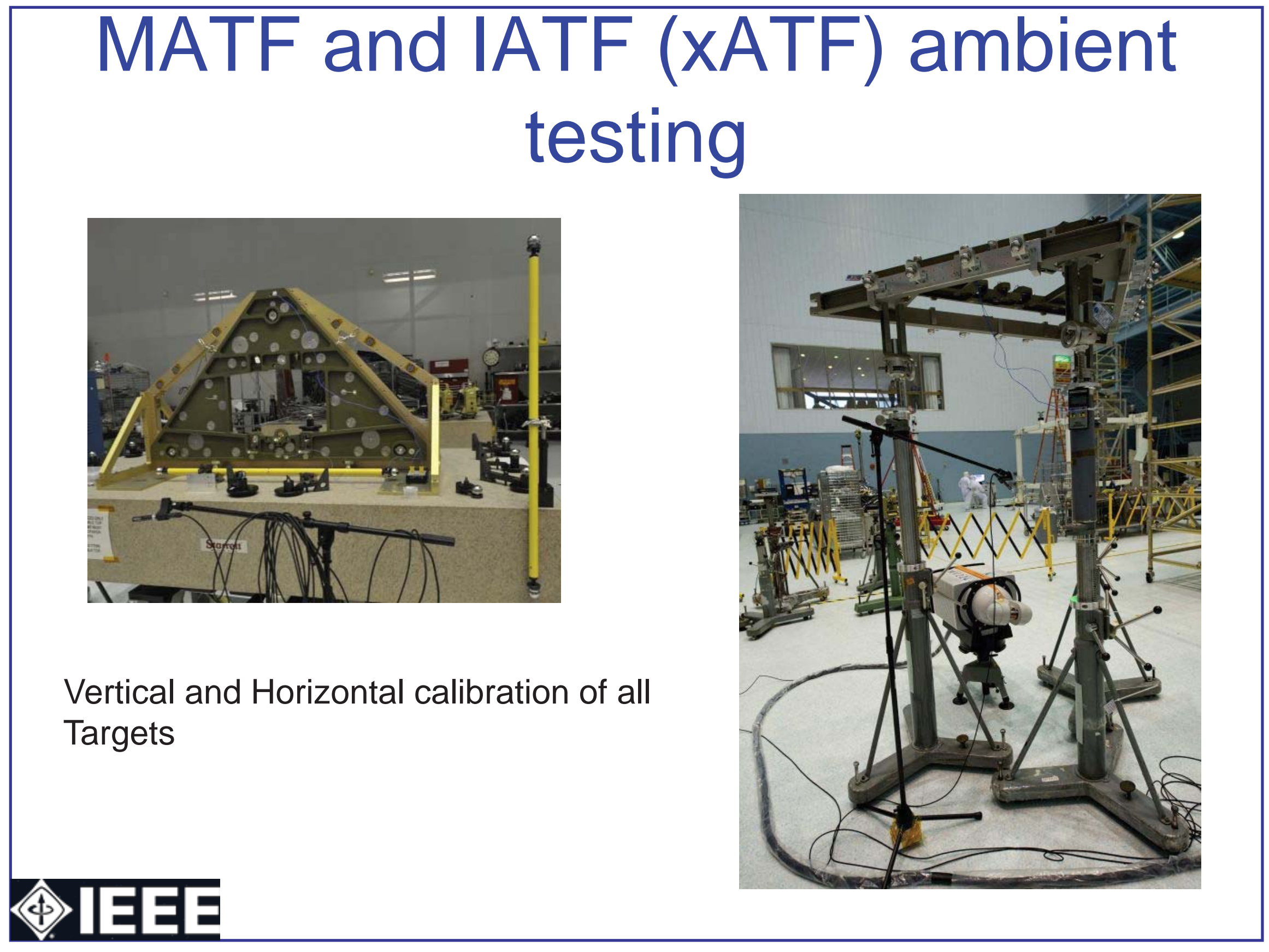




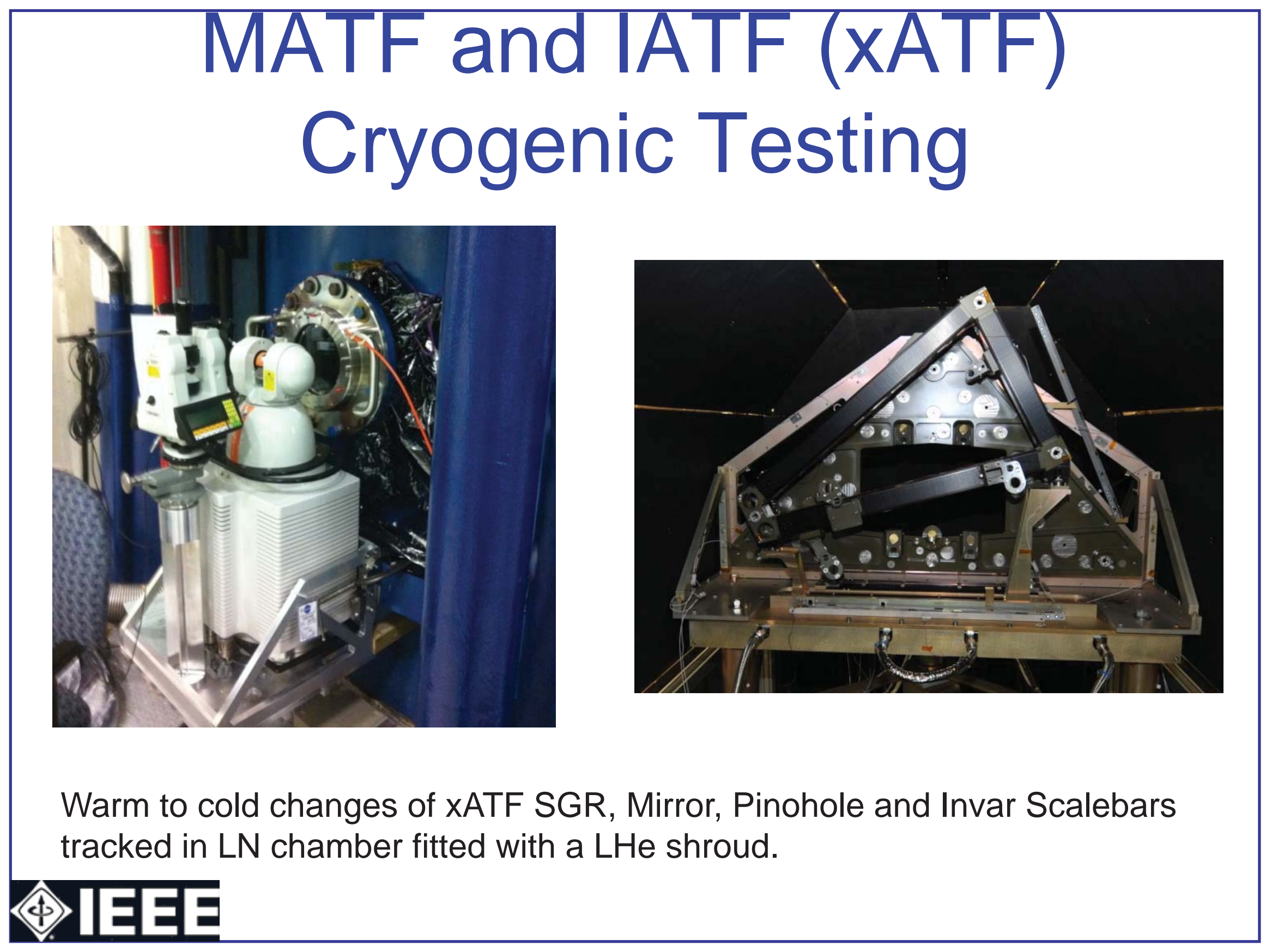




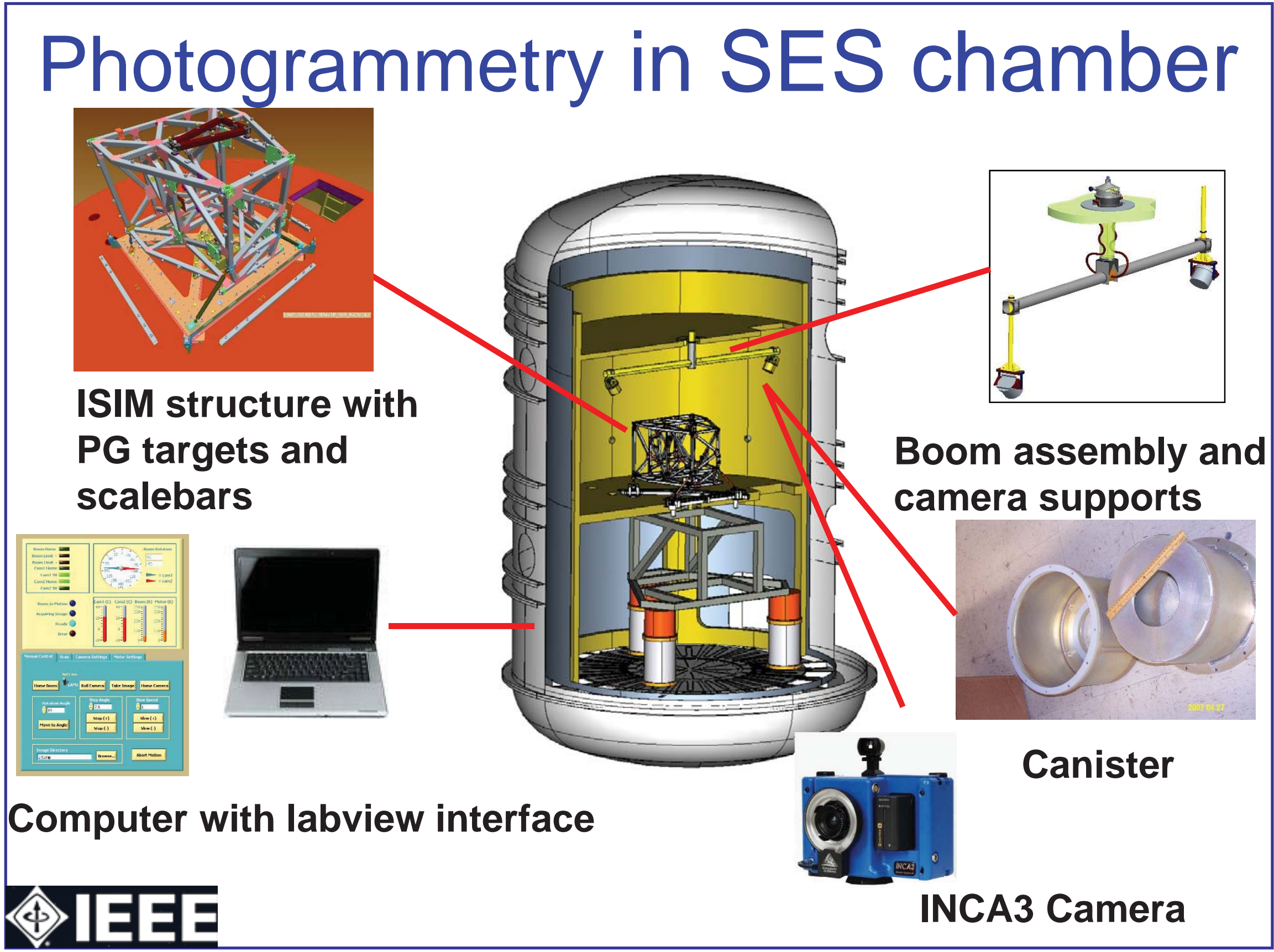




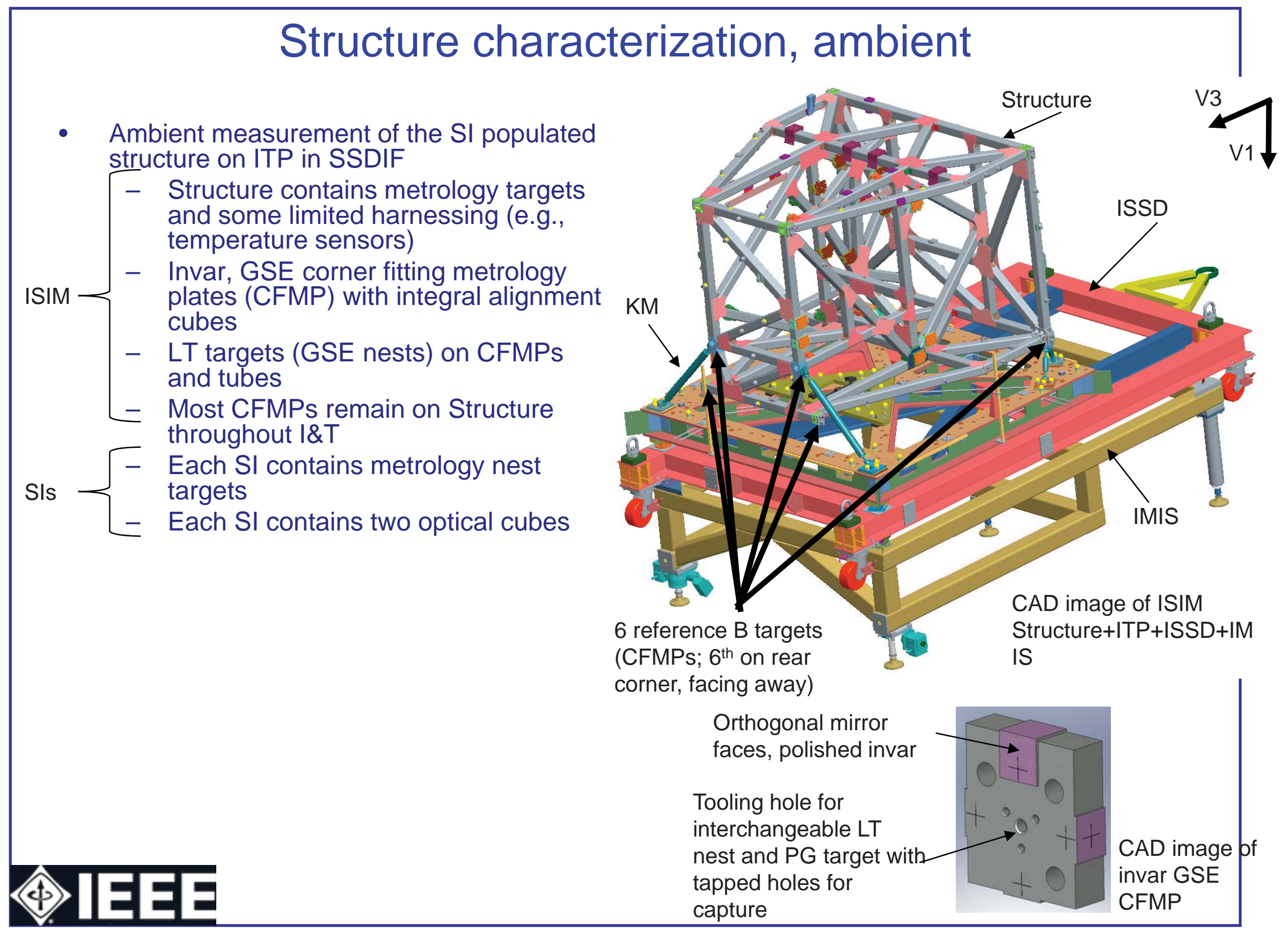




\section{Top View of Test Setup}

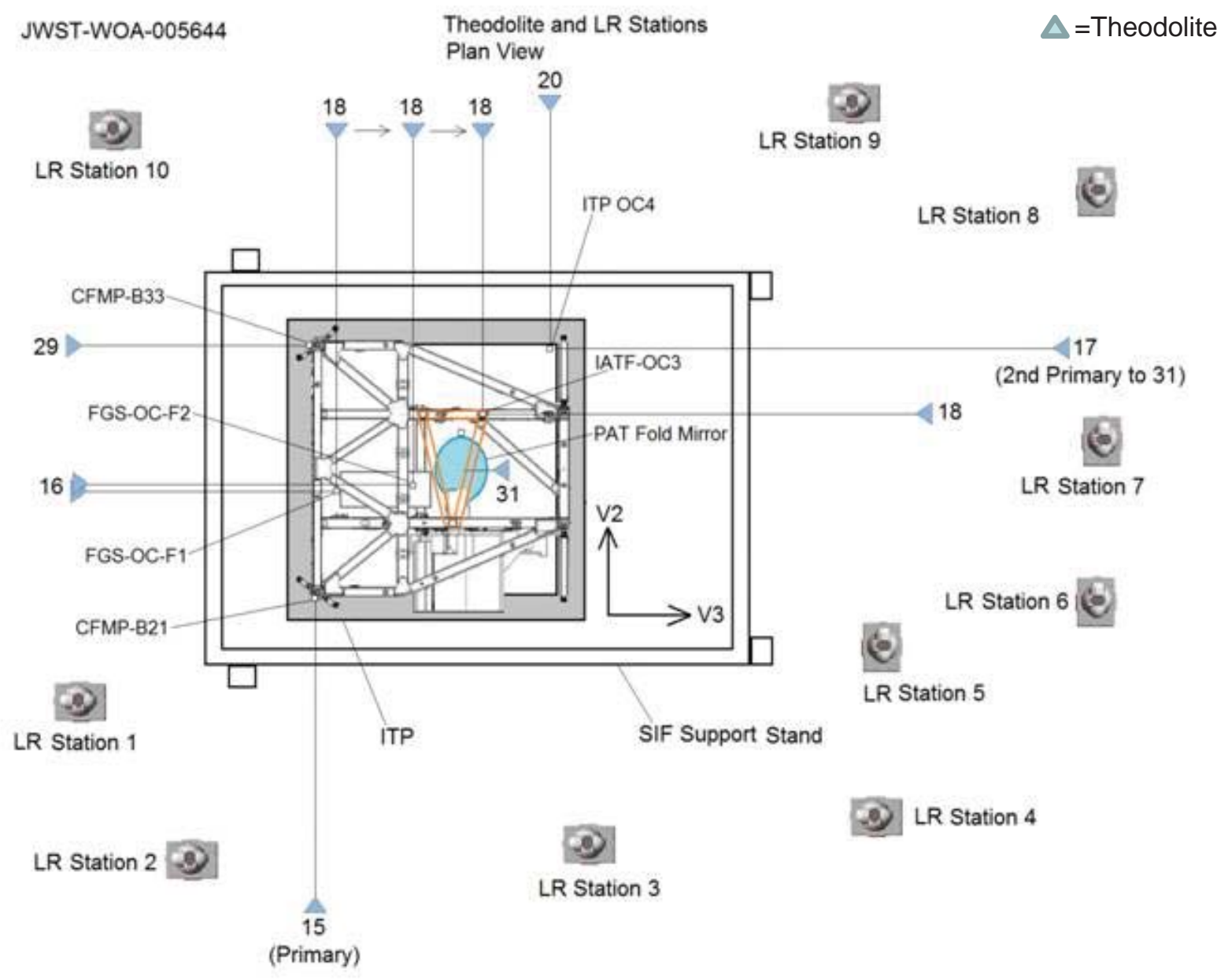

Sls not shown 


\section{PAR Measurement Setup}

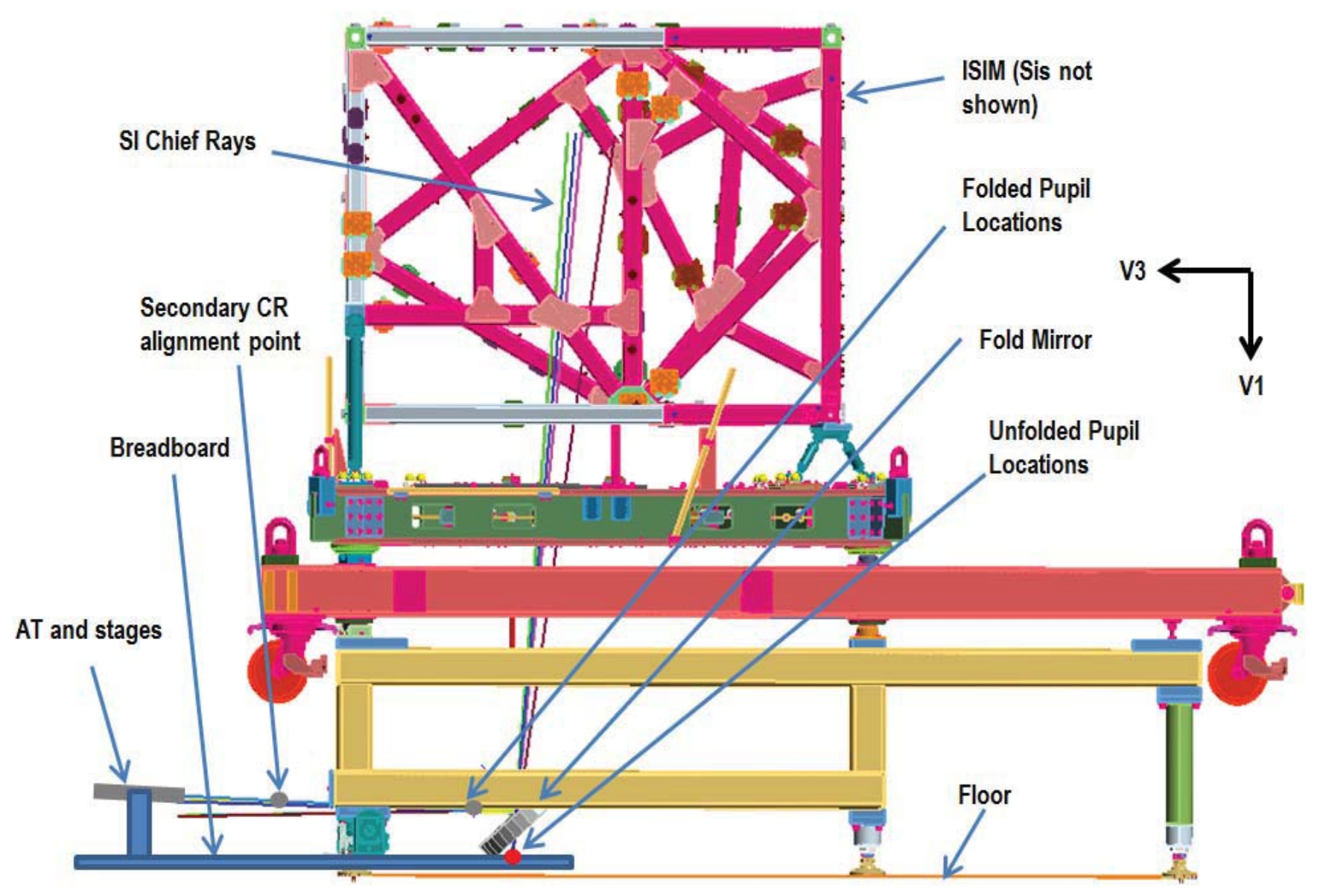




\section{PAR Breadboard Top View}

Breadboard

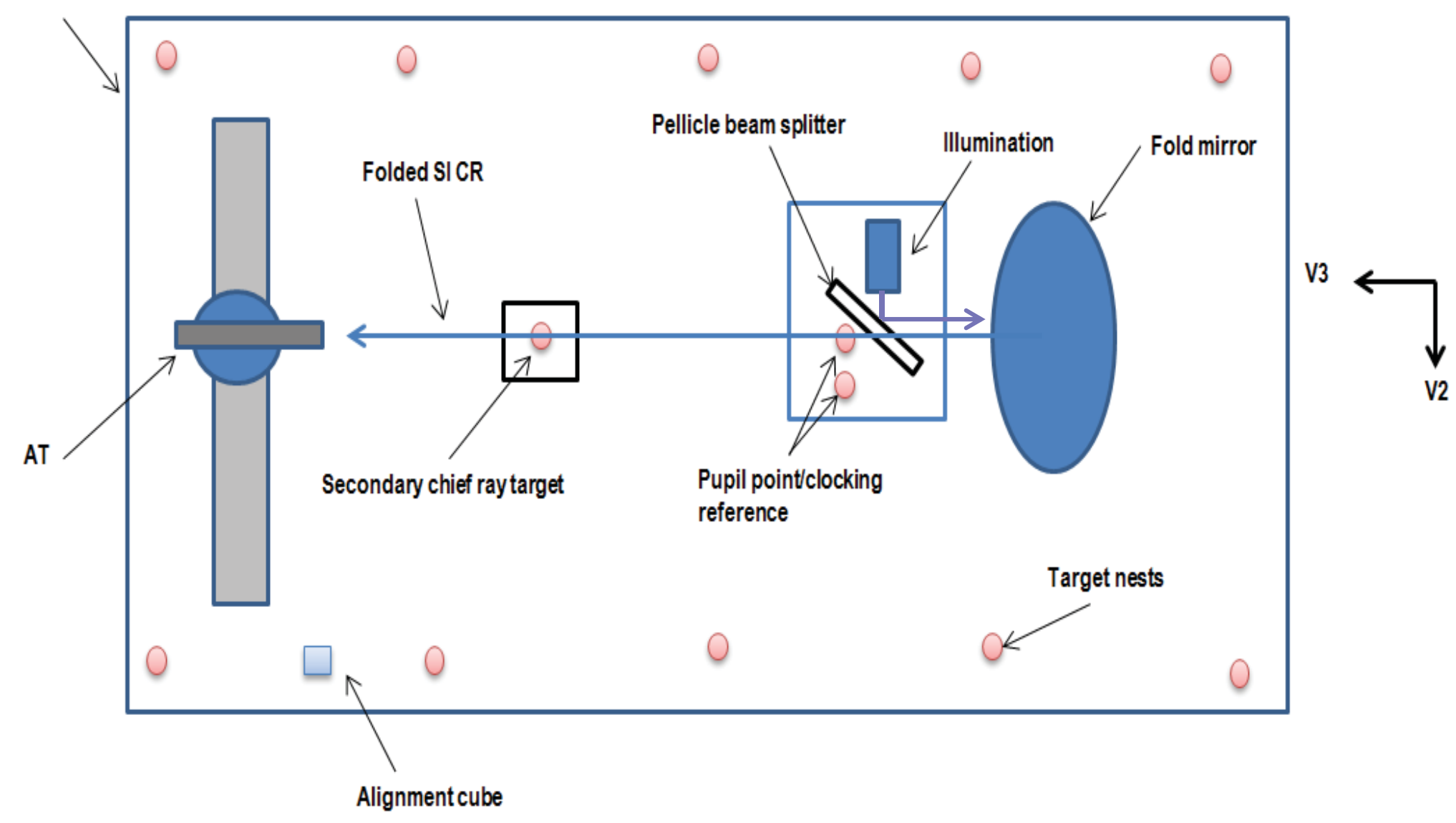




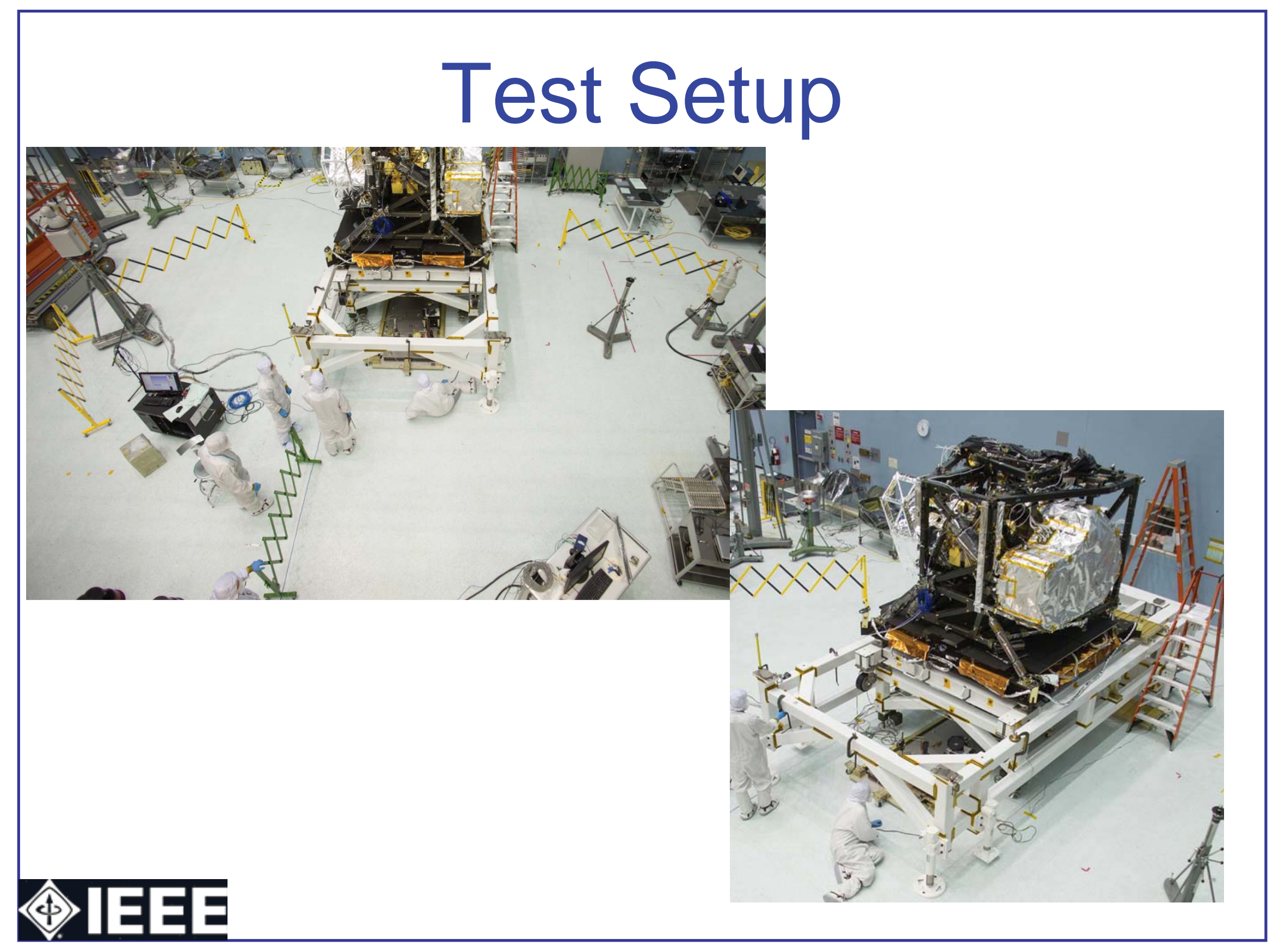




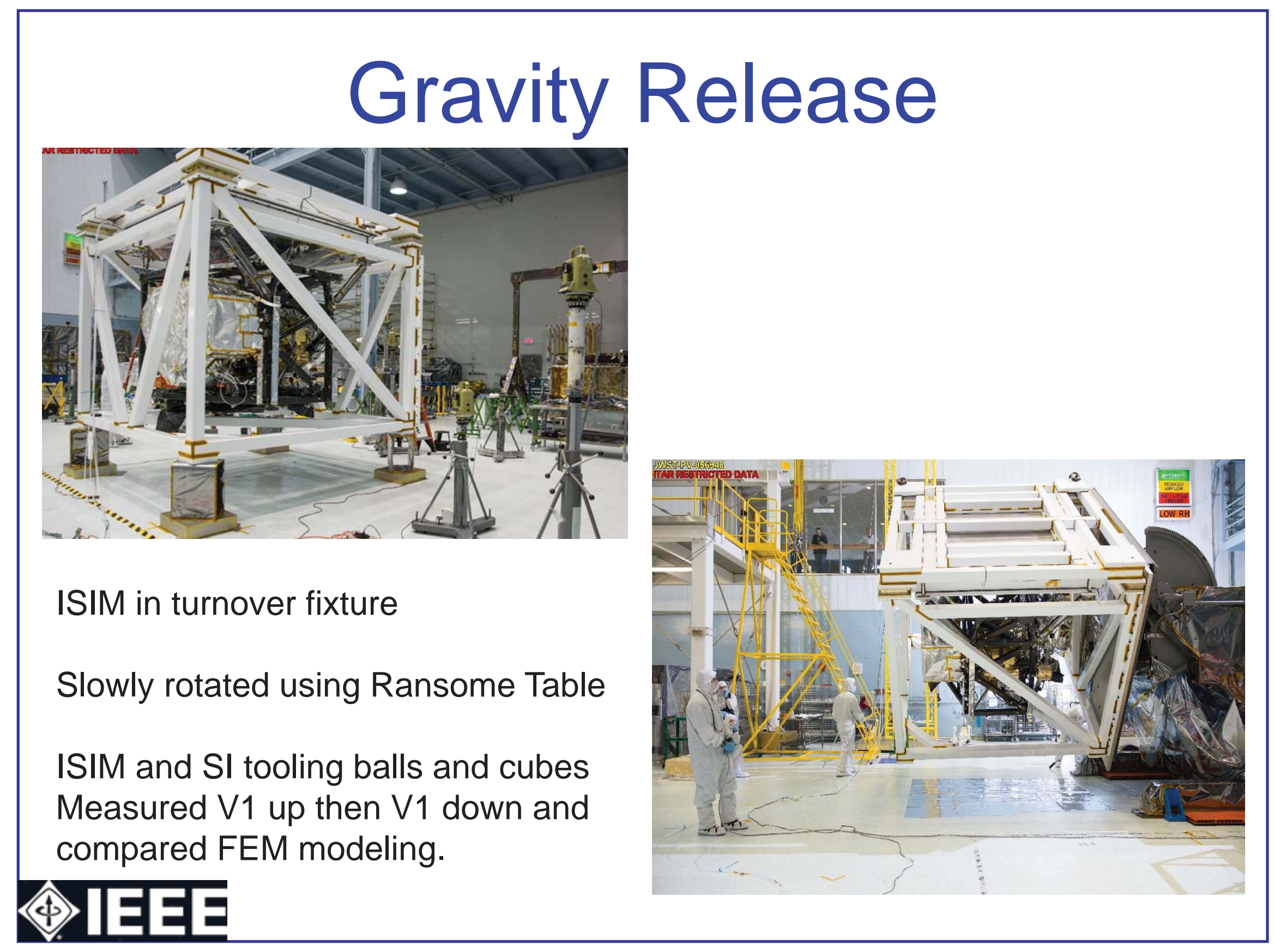




\section{Analysis}

- TB/SMR Data analyzed using Spatial Analyzer ${ }^{1}$

- PG Data analyzed with VStars

- Multiple stations were combined using USMN2,3

- Bundling technique similar to photogrammetry applications

- Can be used for multiple types of instruments

- ISIM nest target values best-fit transformed to an as-built unpopulated ISIM structure database

- Theodolite data analyzed using Microsoft Excel

- Theodolite data brought into VCS via direct and through measurements using the transfer cube assembly (TCA)

- Students-t (2-sigma) ${ }^{4}$ uncertainty calculated from five sets

- FEM differences from gravity are accounted for in the results

- Differences in the coordinate system due to the SIIP from ASMIF to ISIM are accounted for in the results

1. New River Kinematics, Inc., Williamsburg, Va.

2. S. Sandwith \& R. Predmore, "Real-time 5-micron Uncertainty with Laser Tracking Interferometer Systems using Weighte Trilateration," New River Kinematics, Inc. Williamsburg, Va.

3. Spatial Analyzer User Manual, page 322, v. 2013.12.09. 


\section{GSE Targets: Pinholes}
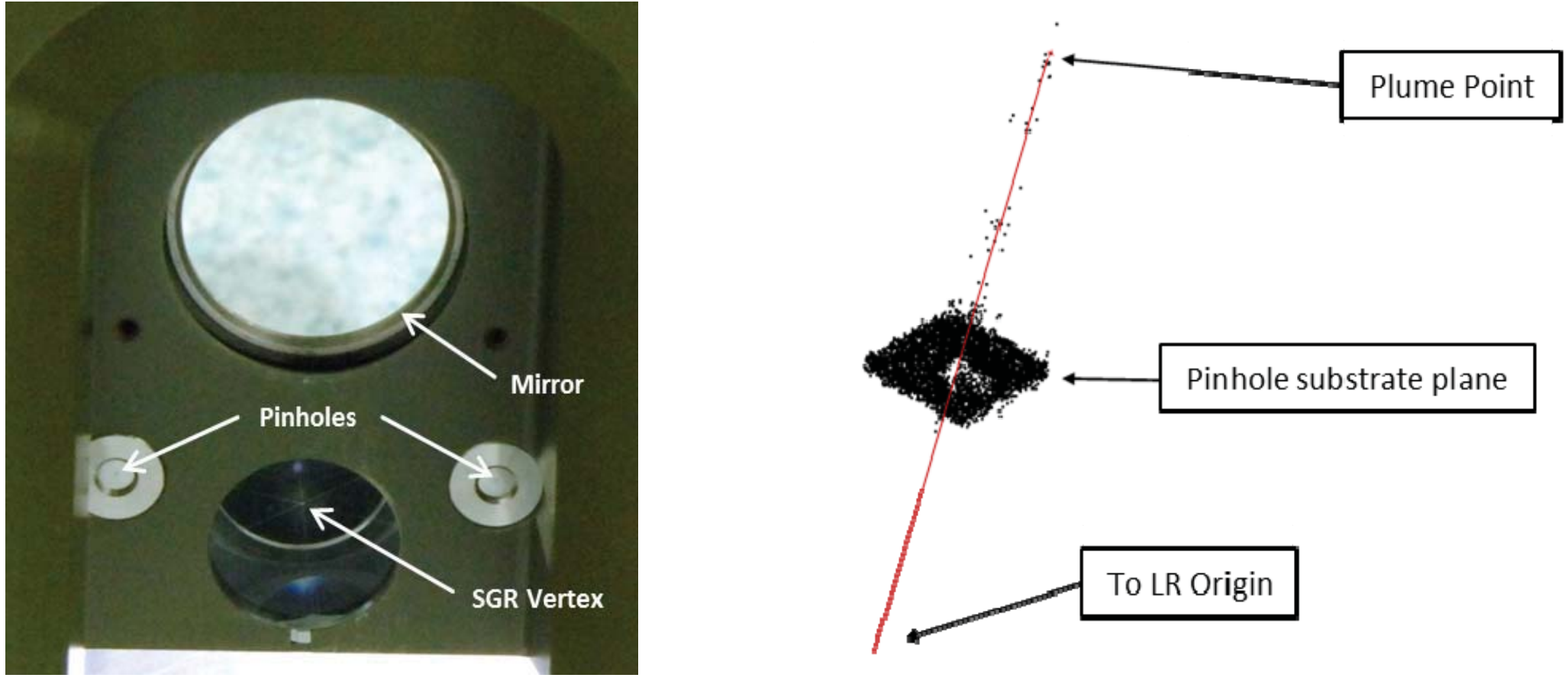

Ambient Pinhole measurements with Cathetometer Cryogenic with LR vision scan(output pictured above) 


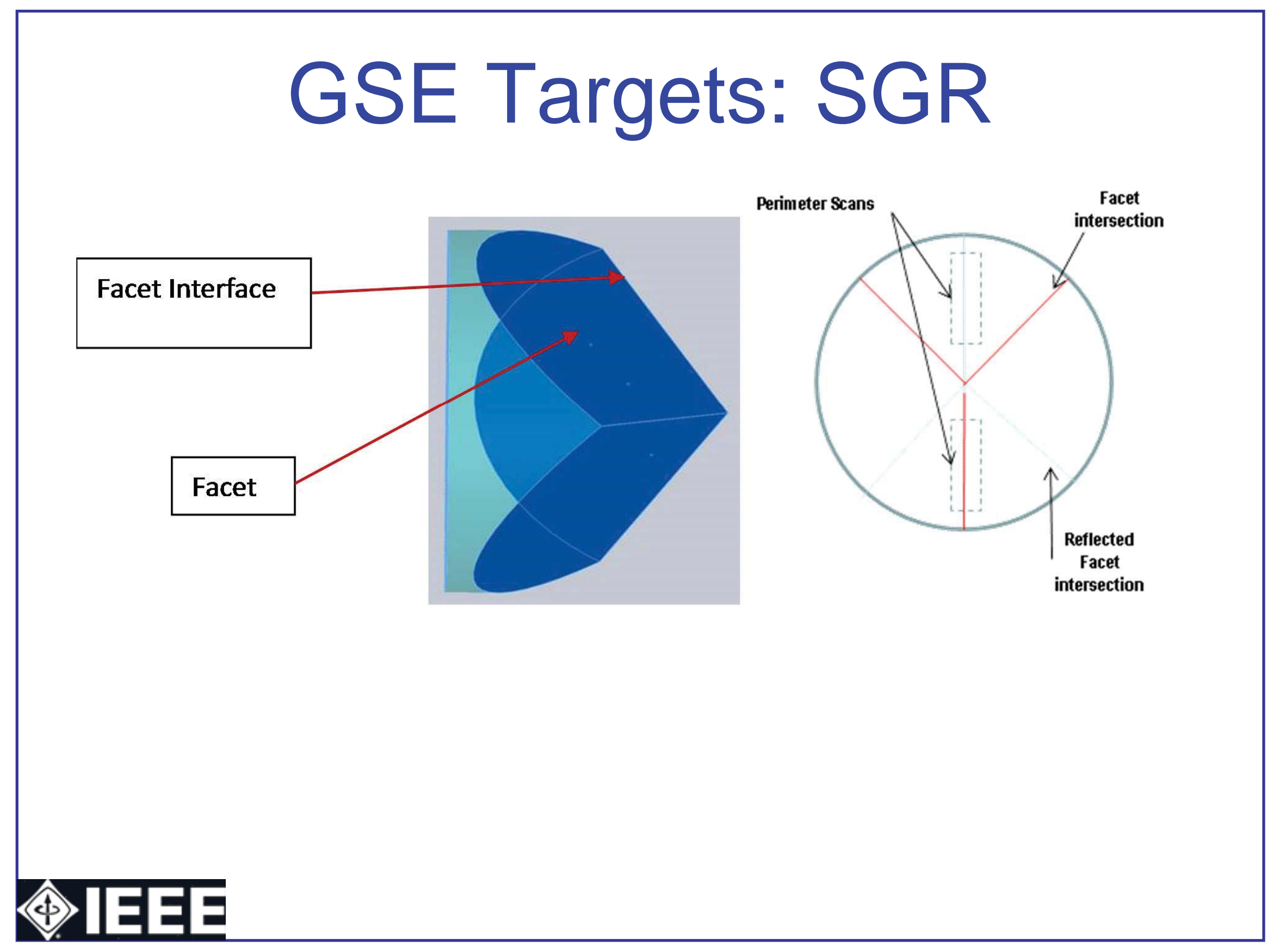




\section{GSE Targets: SGR}
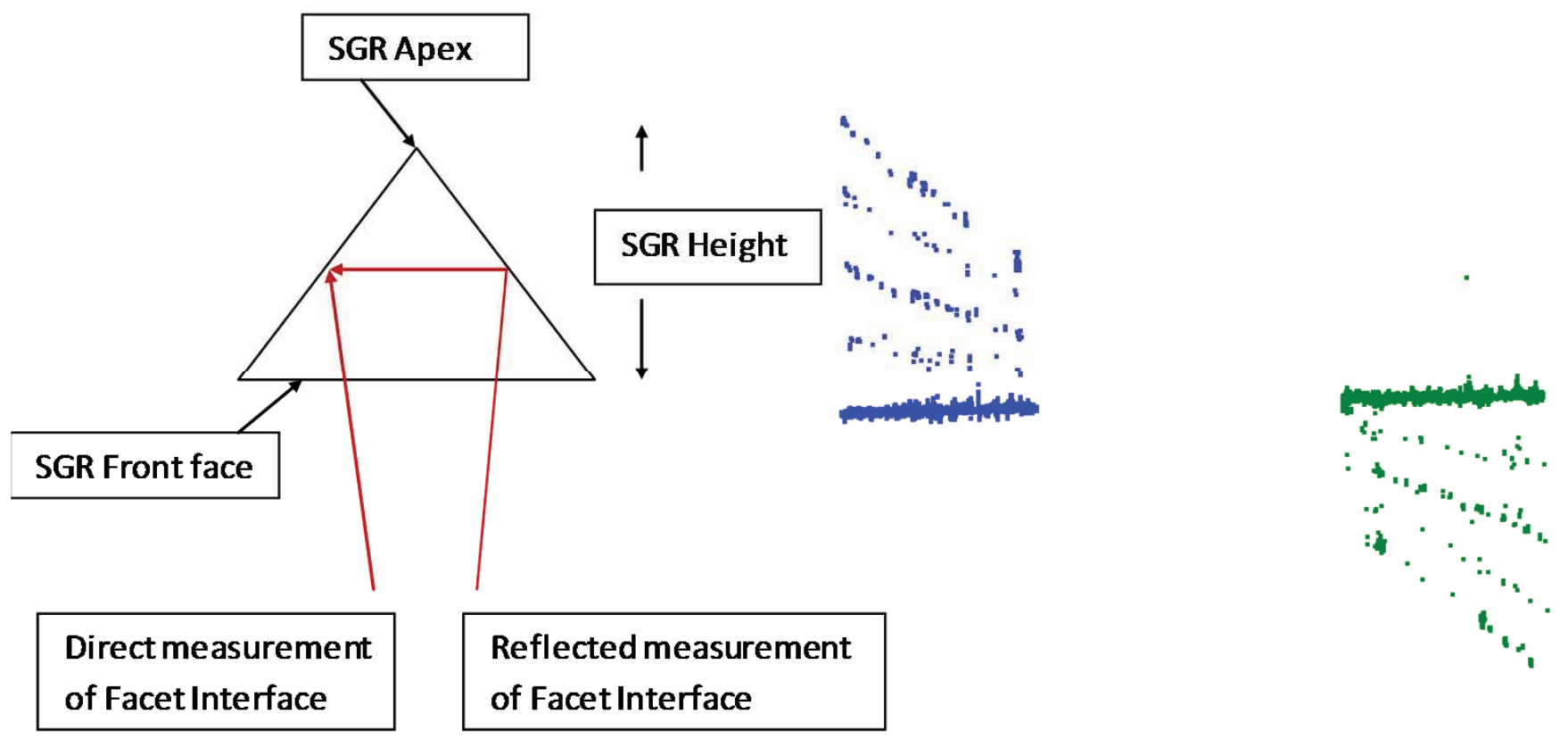

Diagram and data from LR scan of an SGR 


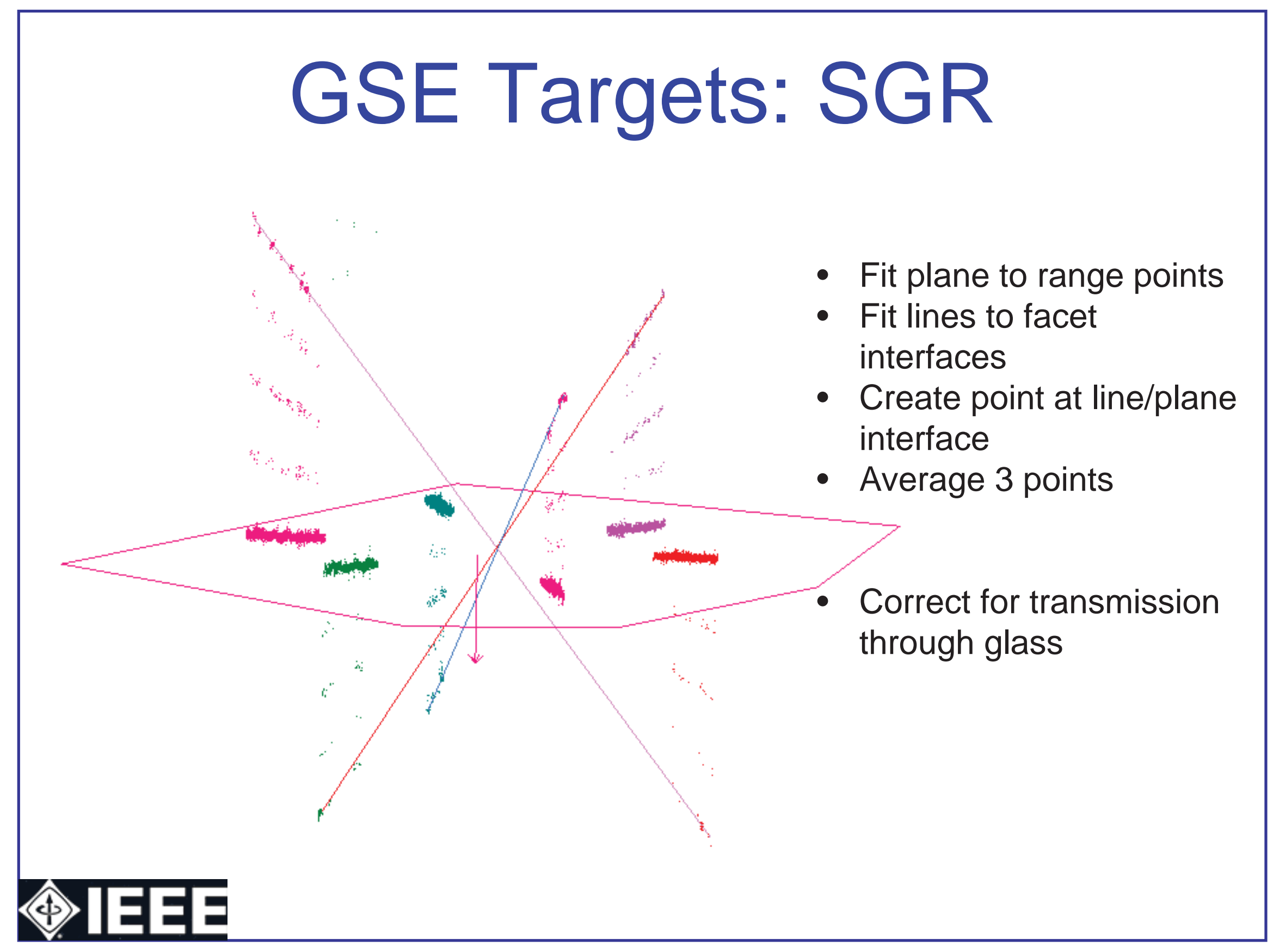




\section{PAR Analysis}

- Analysis starts with the final USMN average results from the TB/SMR survey of the structure

- Measurements are made of all visible ISIM structure targets during the PAR measurements and are best-fit transformed to the final USMN results of the ISIM survey

- The measured pupil target location is used as the basis for image analysis

- ImageJ software is used for the image analysis.

- Five images taken with illumination altered between images for each PAR

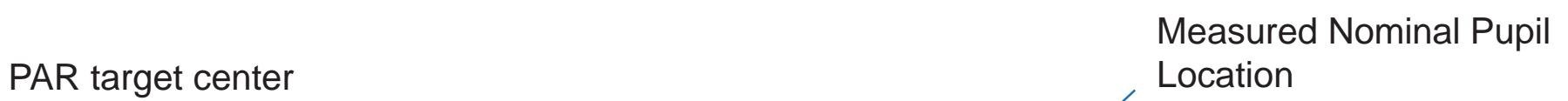

Location
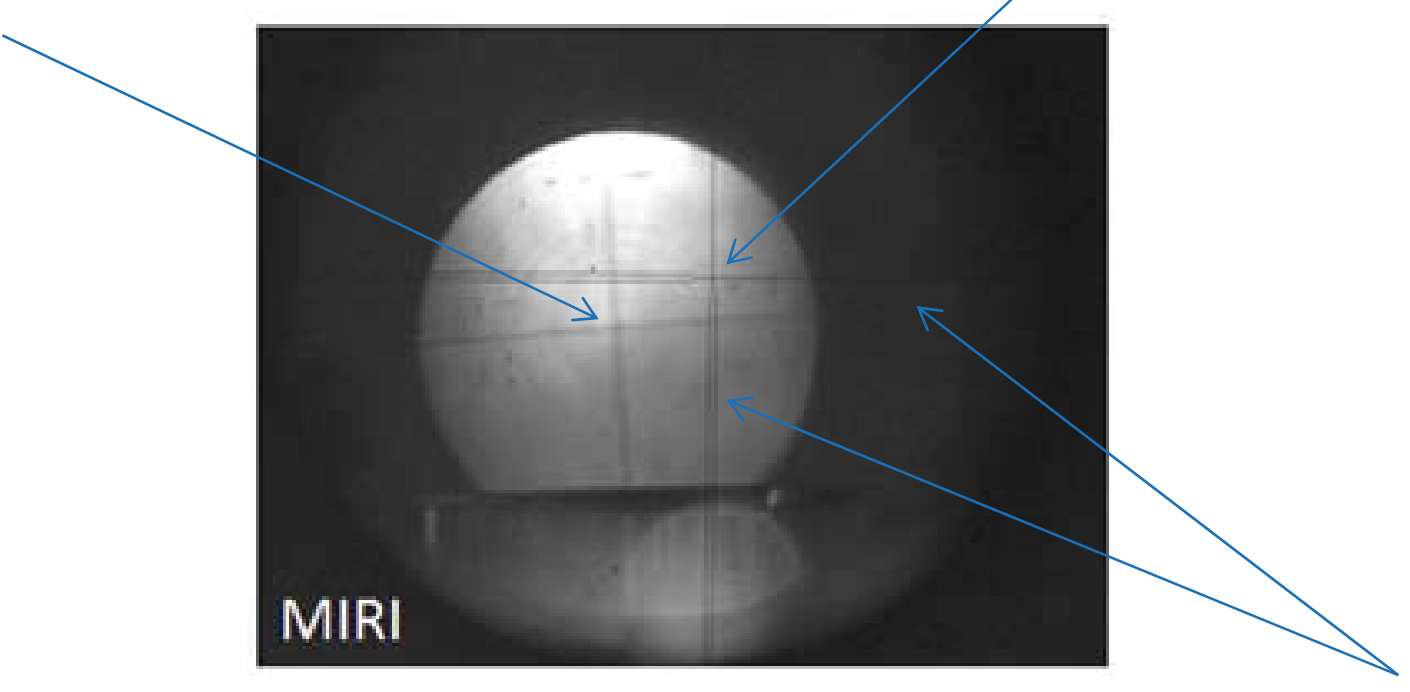

AT crosshairs aligned to V2/V3 


\section{PAR Analysis}

- PAR images from all instruments

- PAR targets are not perfectly aligned to the SI pupil. The offsets are known

- All SIs are not designed to image well at ambient
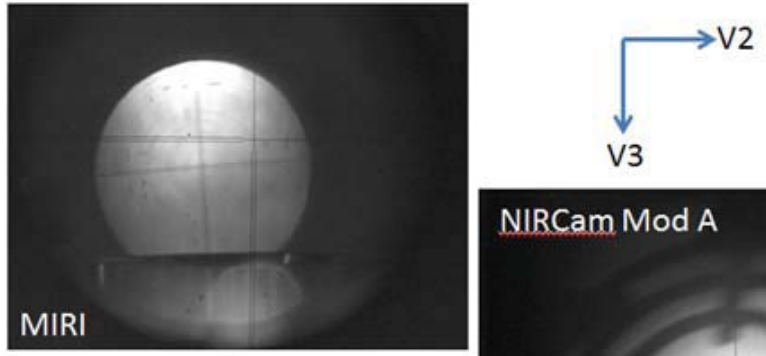

NIRCam Mod A
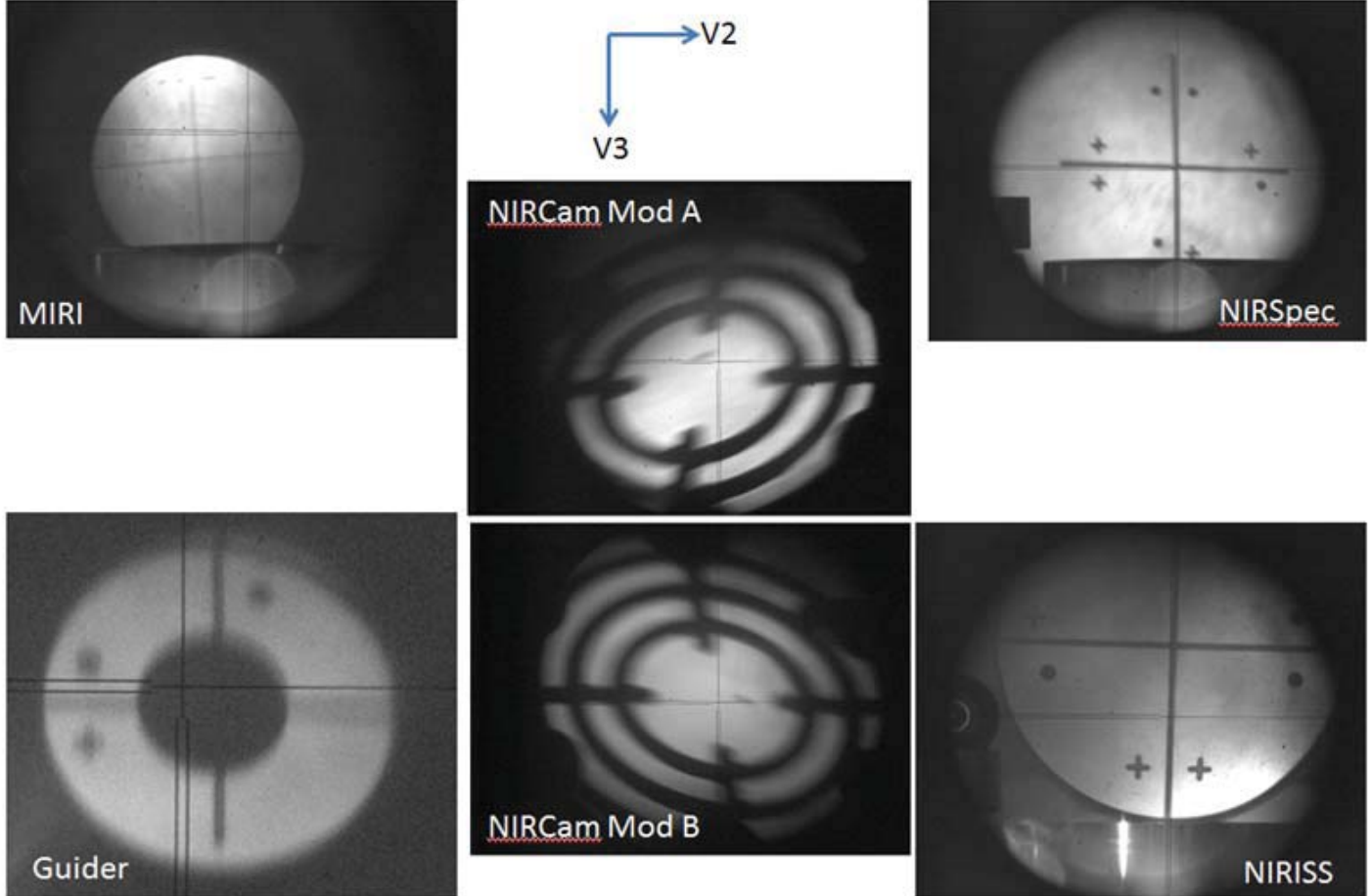


\section{Results}

- ISIM structure data presented is from the pre-cryovac 2 testing (May 2014)

- Pre-CV1 prime-FGS, MIRI

- Pre-CV1-FGS, MIRI

- Post-CV1-FGS, MIRI (PAR only)

- Pre-CV2 prime-All SIs

- Pre-CV2-All SIs

- Post-CV2 (Fall 2014)_All Sls

- FEM difference due to the different SI orientation and loading conditions are accounted for in the results. 


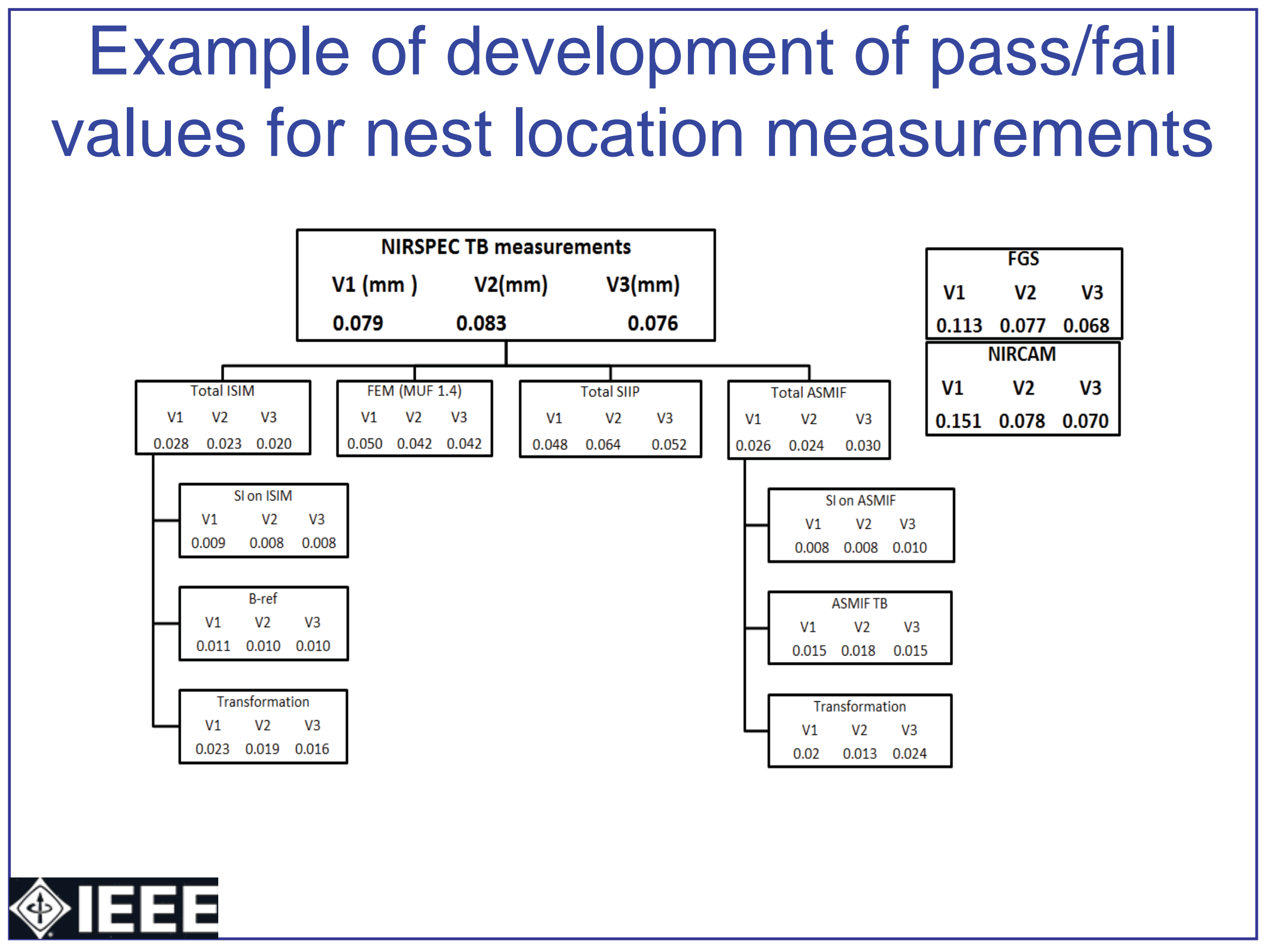




\section{Example of development of pass/fail values for cube face orientation measurements}

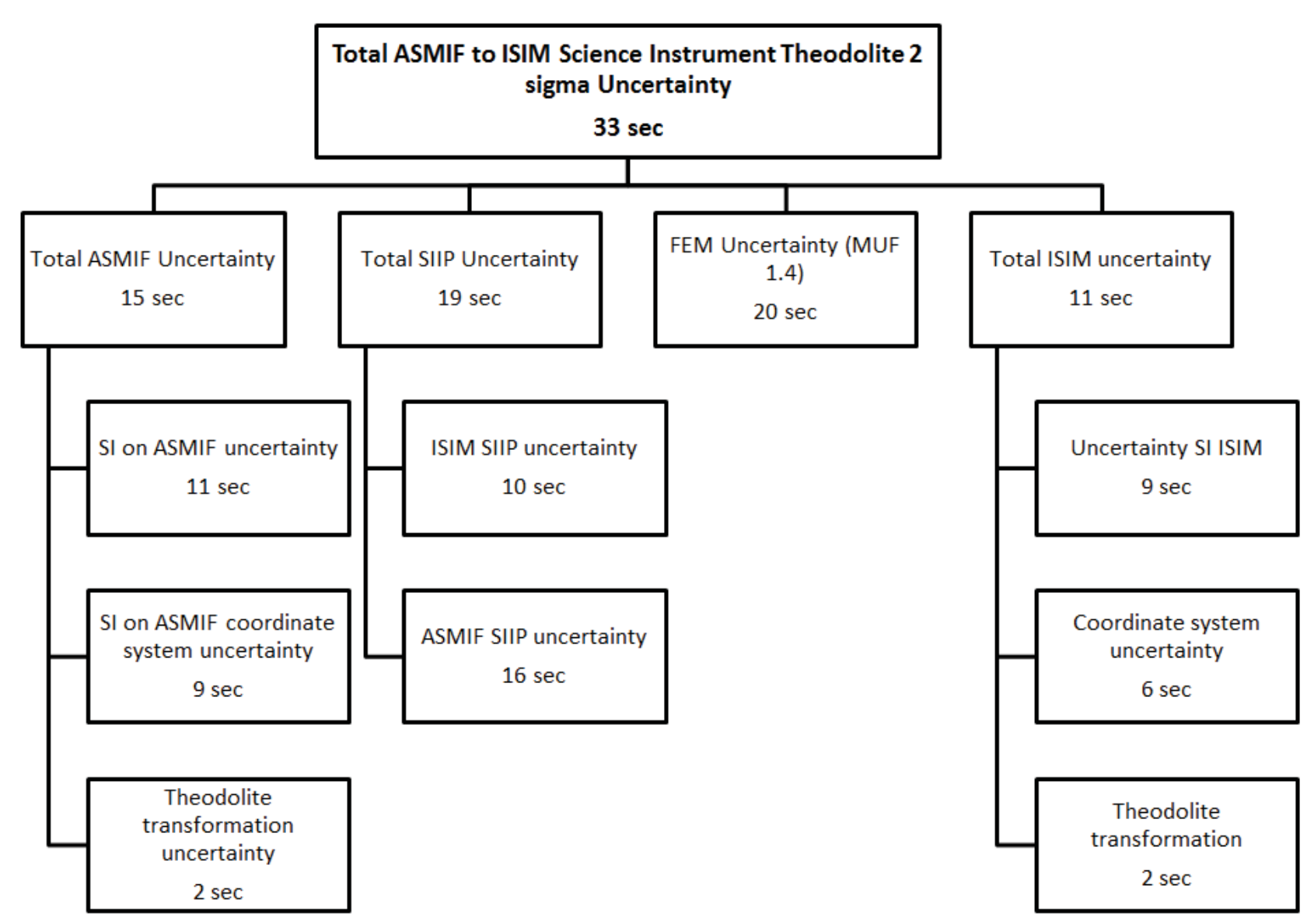




\section{PAR Image Location Pass/Fail Criteria}

\begin{tabular}{|l|l|r|r|}
\hline \multirow{2}{*}{ Test to test uncertainty $(2 \sigma)$} & V1 $(\mathrm{mm})$ & $\mathrm{V} 2(\mathrm{~mm})$ & $\mathrm{V} 3(\mathrm{~mm})$ \\
\cline { 2 - 4 } & 0.071 & 0.239 & 0.239 \\
\hline
\end{tabular}

- Based on relative test to test changes

- Defined in the entrance pupil space

- ISIM level requirement for pupil shear is 3.1\%

- To put this into perspective the pass fail values for V2/V3 converted to pupil shear percent is $0.16 \%$. This is a factor of 20 better than the absolute alignment requirement for pupil shear

$$
\% \text { Pupil Shear }=100 \frac{\Delta V}{152} m m
$$

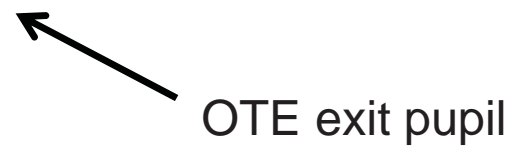




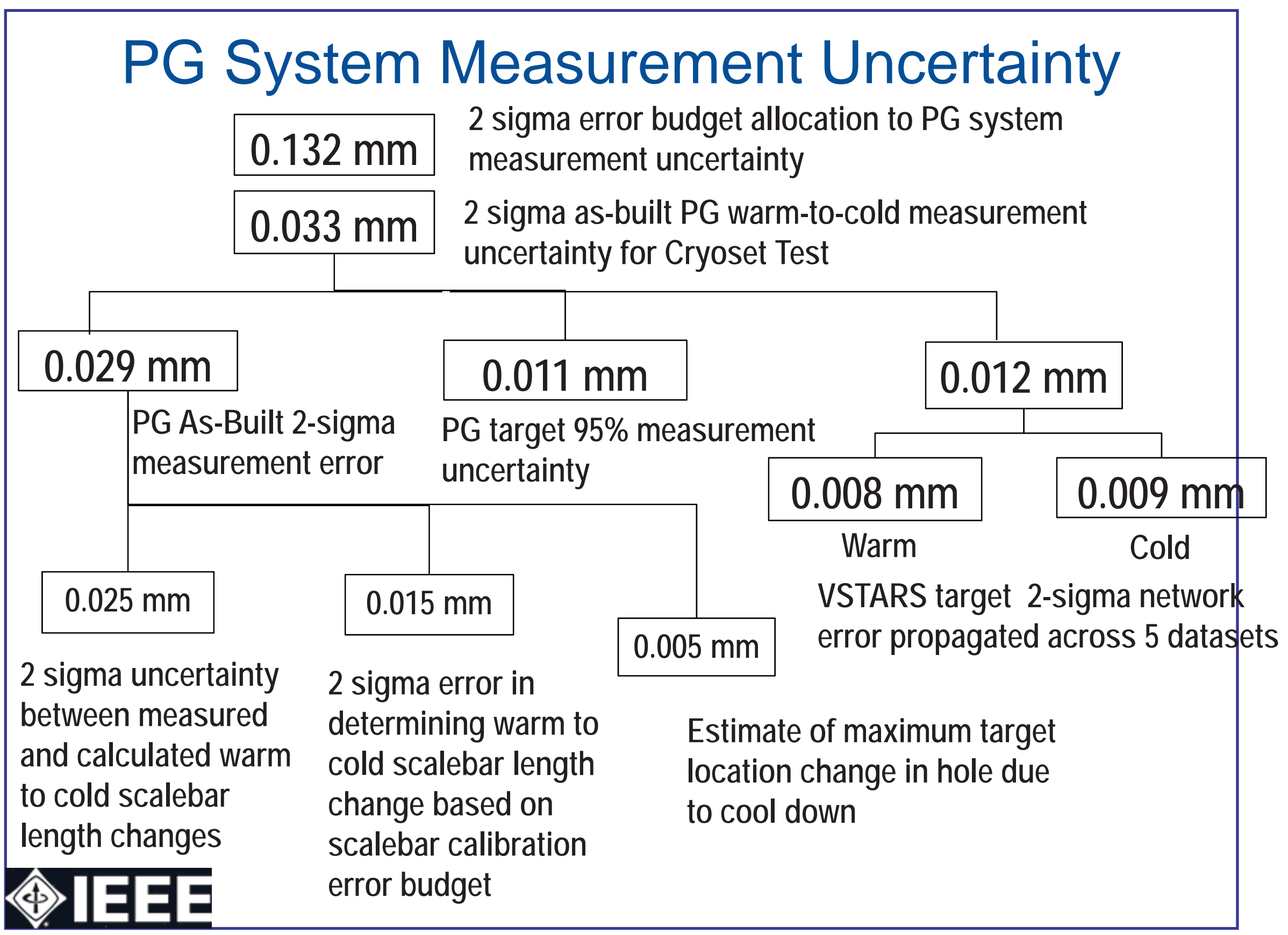




\section{ISIM Coordinate System}

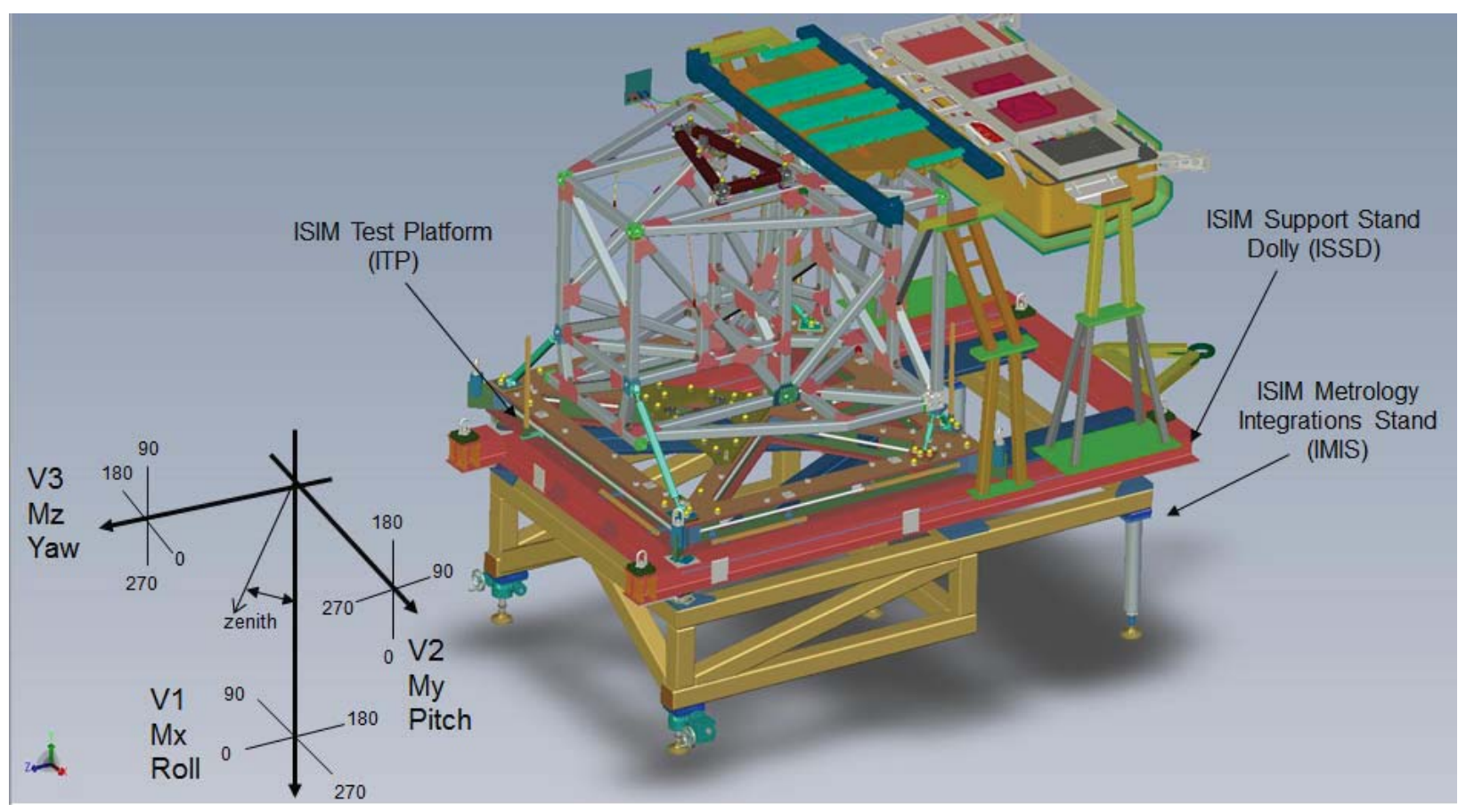




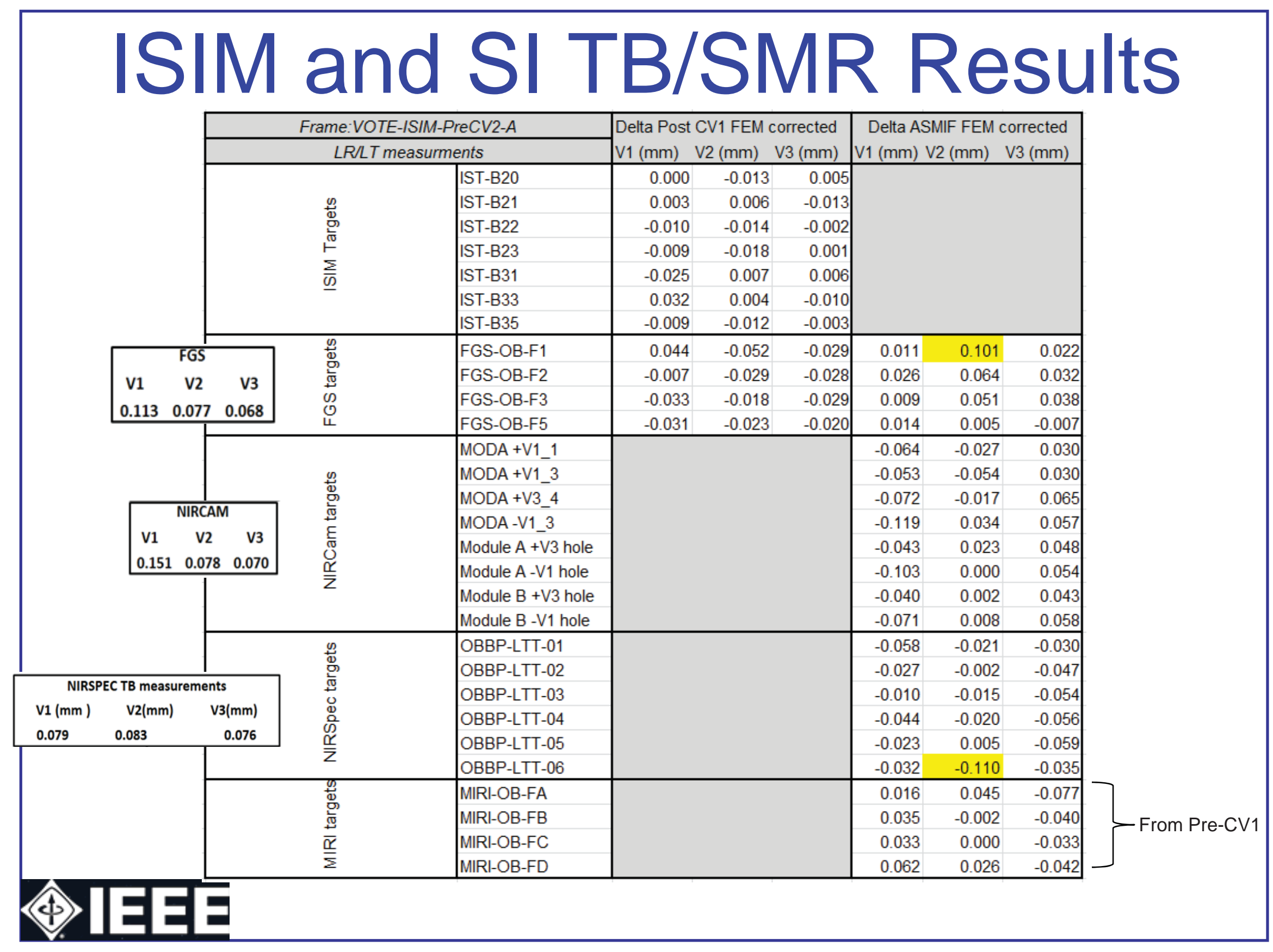




\section{ISIM/SI Cube Results}

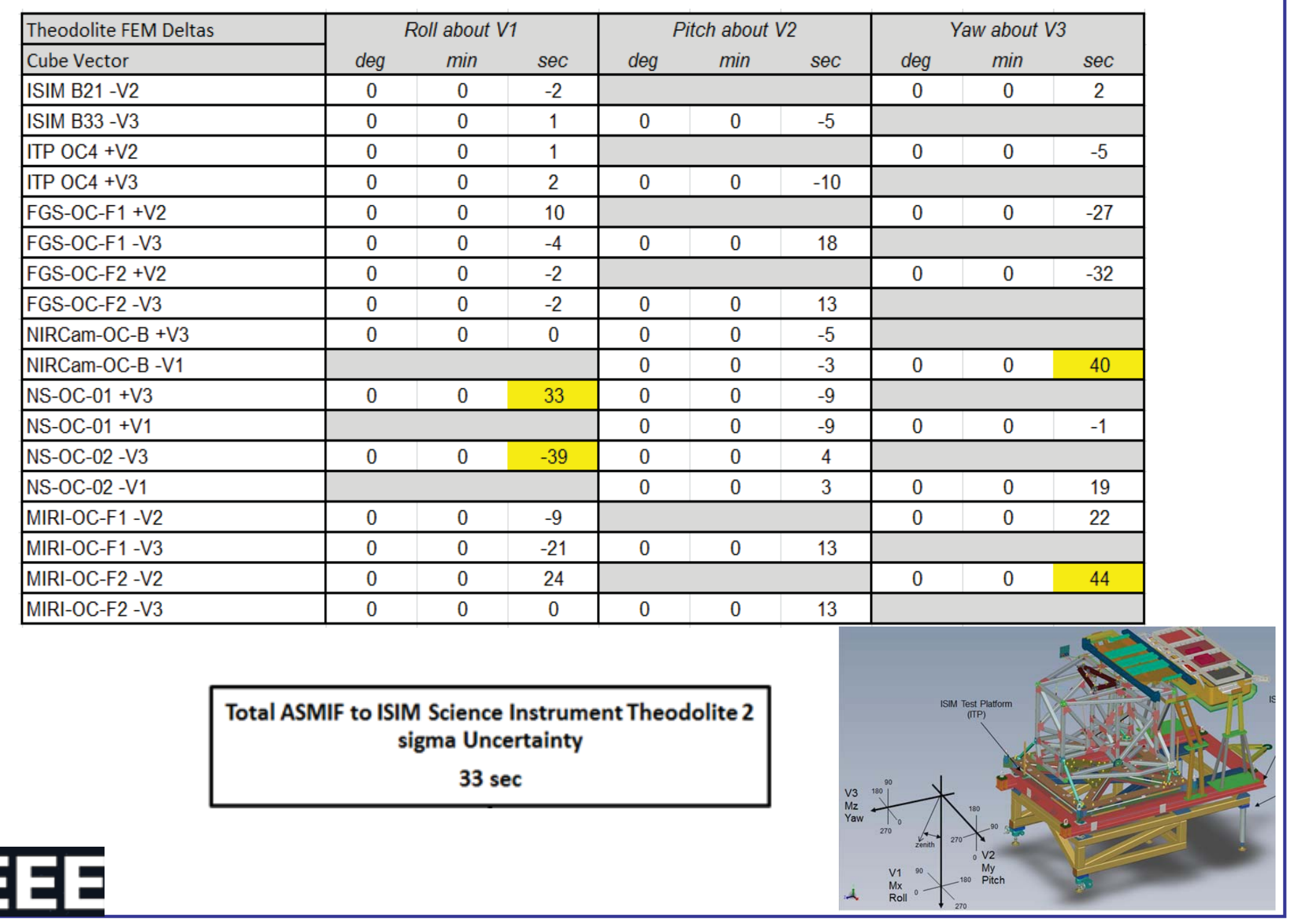




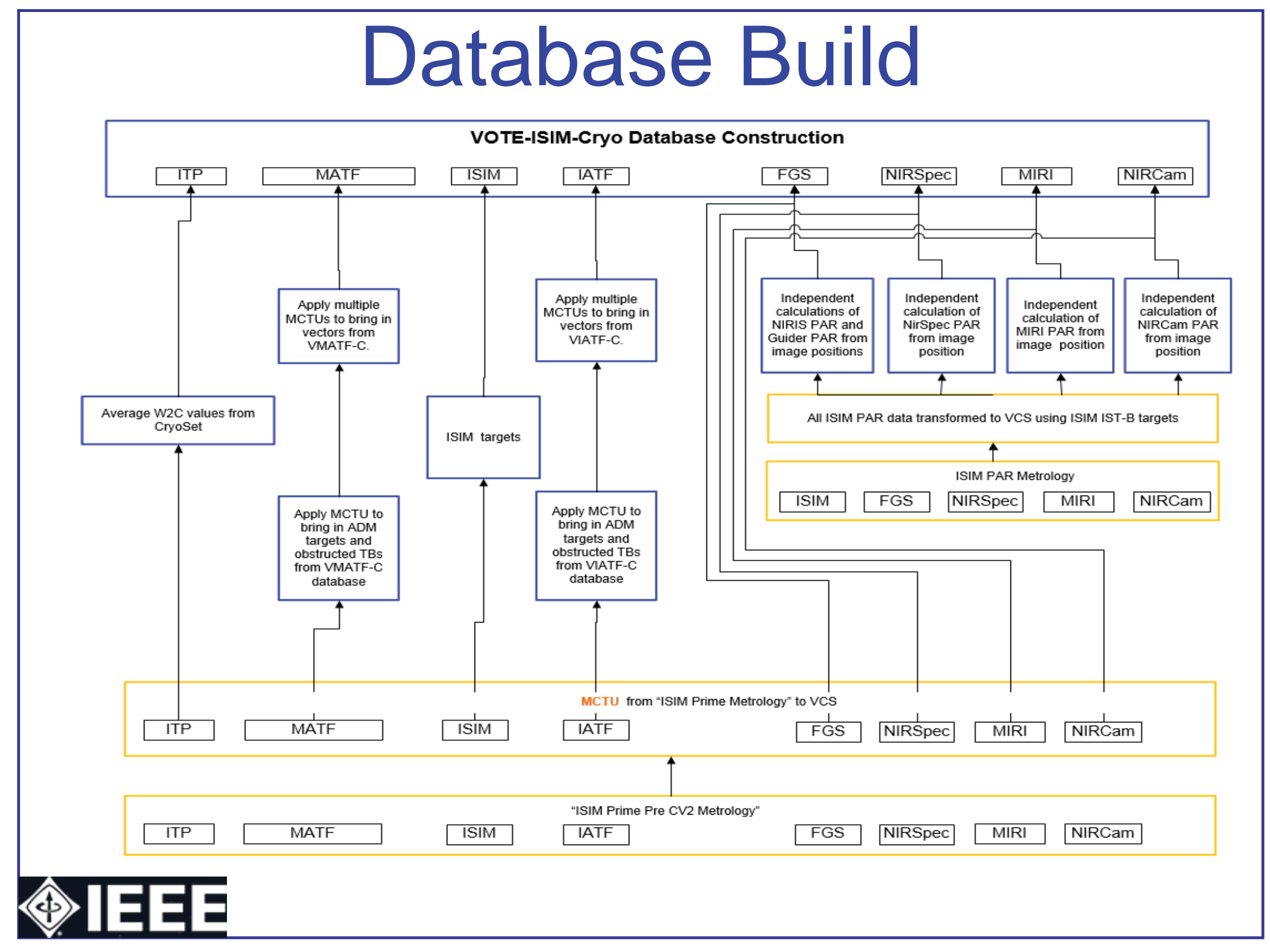




\section{Summary}

- We have successfully verified the SI-level target calibration is in good agreement with measured SI OB locations on ISIM element to better than the required pass/fail values

- This process will be repeated during ISIM level I\&T to trend any potential alignment changes due to thermal cycling (CV2, CV3), vibration and acoustic exposure

- This process will also be repeated after SI work during I\&T. 


\section{Acknowledgements}

The author gratefully acknowledges the collective contributions of the optical, mechanical, and systems engineering, management, and science teams working on the James Webb Space Telescope, Integrated Science Instrument Module element, and specifically:

J. Gum, T. Hadjimichael, J. Hylan, T. Madison, L. Miner, R.G. Ohl, J. Young

NASA Goddard Space Flight Center, Greenbelt, Maryland

\section{Maszkiewicz}

Canadian Space Agency

A. Beaton

Comdev International

S. Hummel, M. Melf, A. Roedel

EADS Astrium Gmbh

M. Te Plate

European Space Agency

P. Schweiger

Lockheed Martin Corporation

K.F. Mclean, J. McMann, K. Redman, G.W. Wenzel

Qinetiq North America

J.E. Hayden

Sigma Space Corp., Lanham, Maryland

P.W. Williams

SGT Internationa

D. Lee, M. Wells

UK Astronomy Technology Centre

This work is supported by the James Webb Space Telescope project at NASA Goddard Space Flight Center 


\section{Questions? \\ Thank you for your attention.}

Theo Hadjimichael

Optics Branch

NASA Goddard Space Flight Center

Greenbelt, Maryland

Theodore.j.hadjimichael@nasa.gov tel. 3012865681 


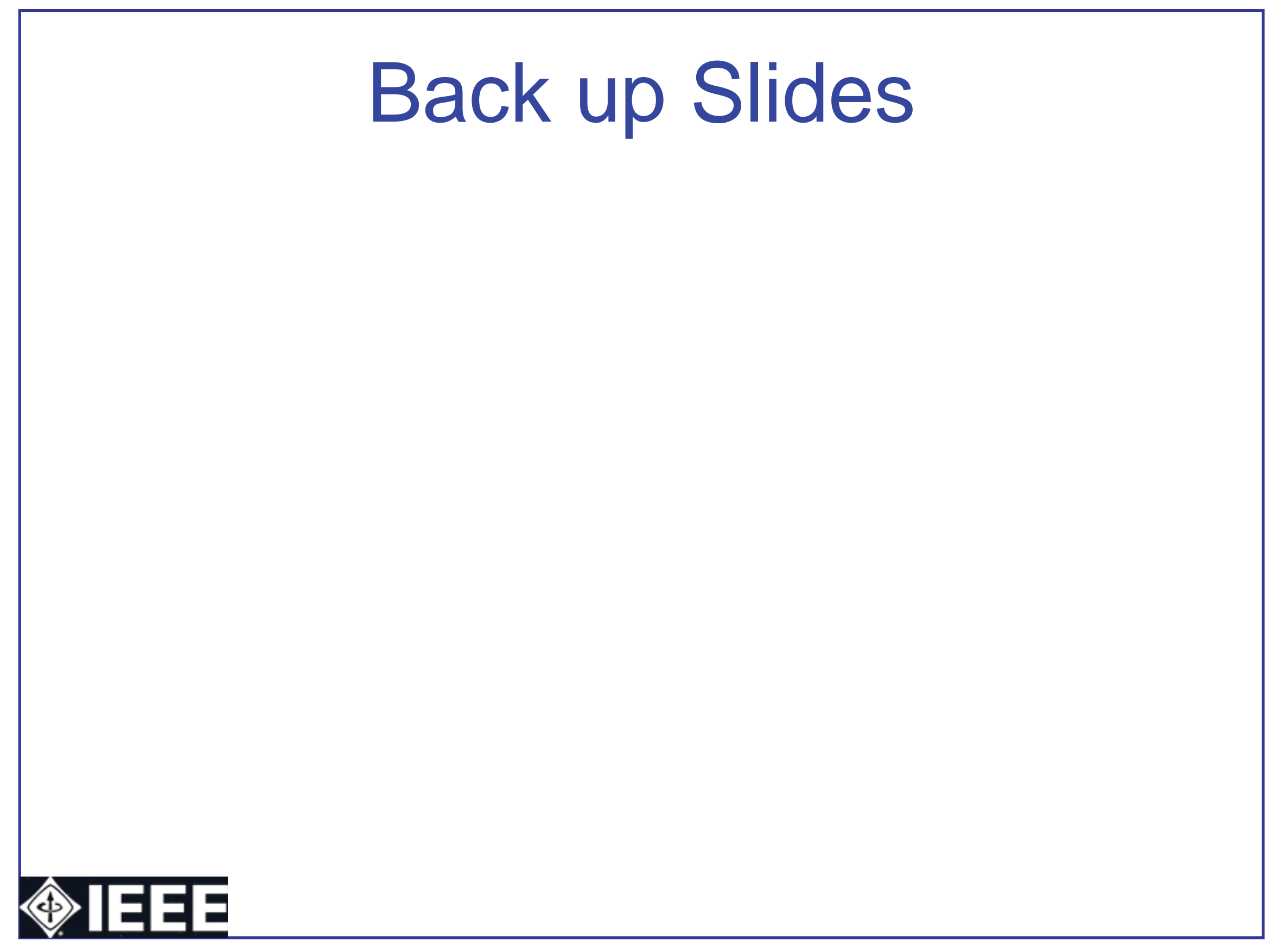




\section{ASMIF status}

- $\quad$ MIRI ASMIF: Delivered to RAL, May 2007

- $\quad$ NIRSpec ASMIF: Delivered to Astrium, Sep 2007

- FGS ASMIF: Delivered to COM DEV, Dec 2007

- $\quad$ NIRCam ASMIF: Delivered to Lockheed Martin, Jan 2009

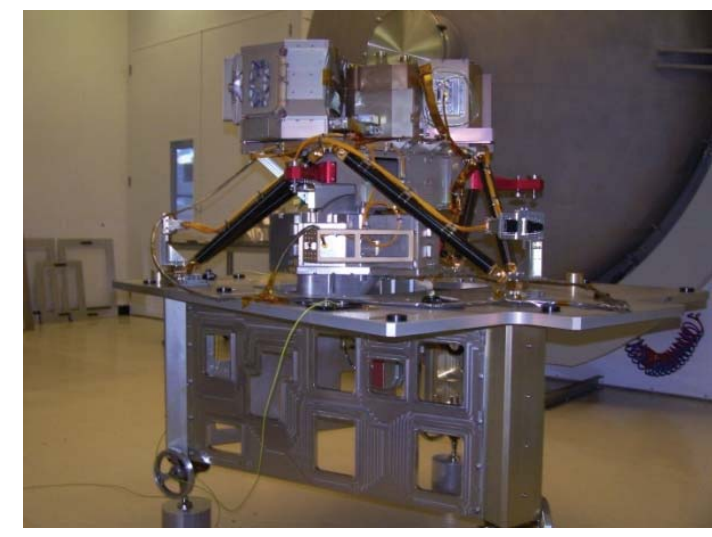

MIRI VM with ASMIF at RAL
FGS ETU with ASMIF at COM DEV, Ottawa
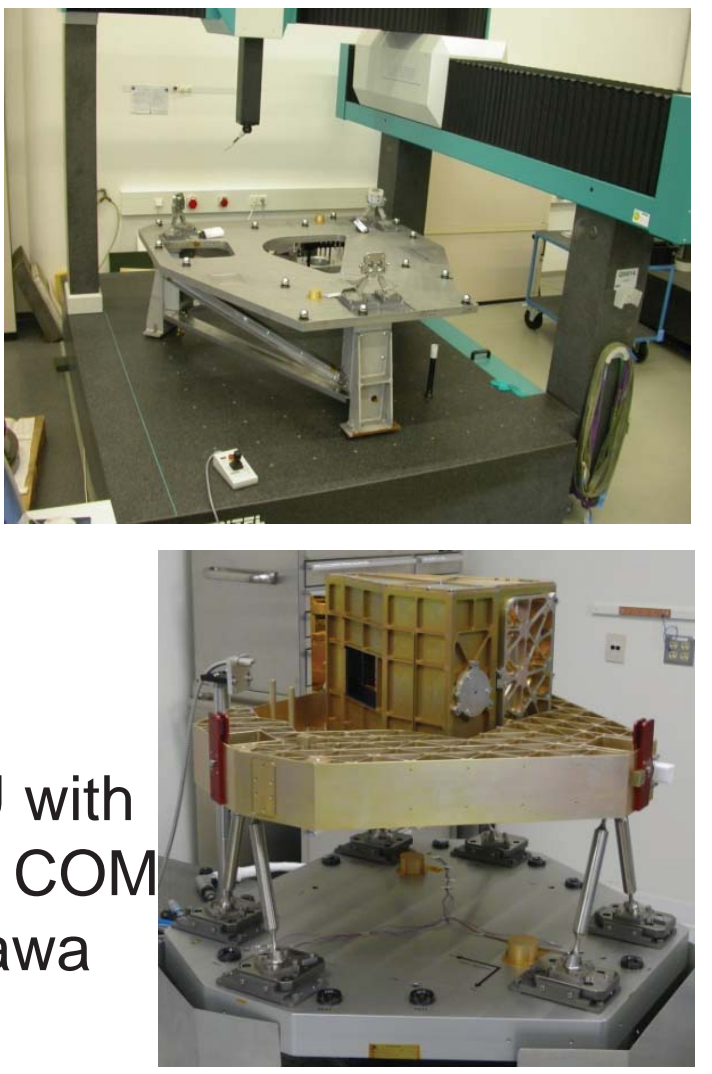

NIRSpec ASMIF postshipment calibration check and OBK installation at Astrium

NC bench installed on ASMIF at LMCO

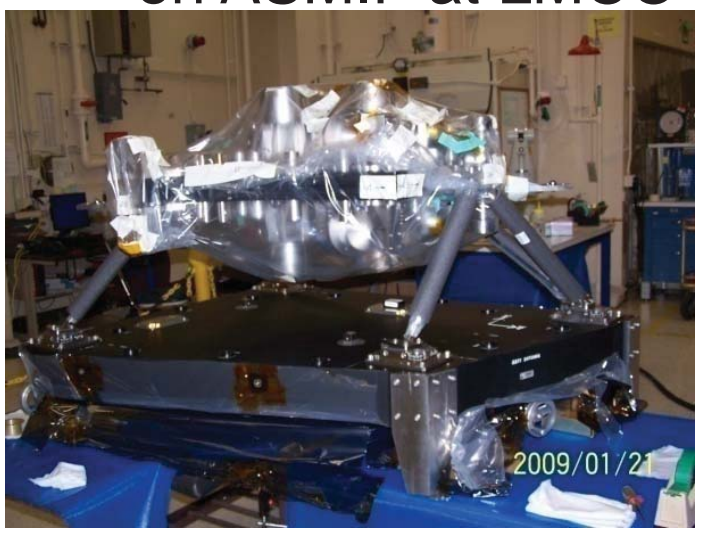




\section{ISIM Test Platform (ITP)}

- Master gauge for nominal OTE-ISIM interface ("reference A")

- Fiduciary points on KM sockets map to MICD

- Used for ambient integration, metrology and alignment (SSDIF)

- Used for cryogenic testing in both Structure cryo-set and Integrated ISIM testing

- Supported by IMIS and ISSD in SSDIF for ambient work

- Supported by Upper GESHA in SES chamber for cryogenic testing

- $\quad-30 \mathrm{~K}$ ITP is attached to the $\sim 100 \mathrm{~K}$ Upper GESHA via thermal isolator stand-offs

- Supports MATF for OSIM-to-reference A alignment

- Extensive optomechanical requirements related to alignment and stability

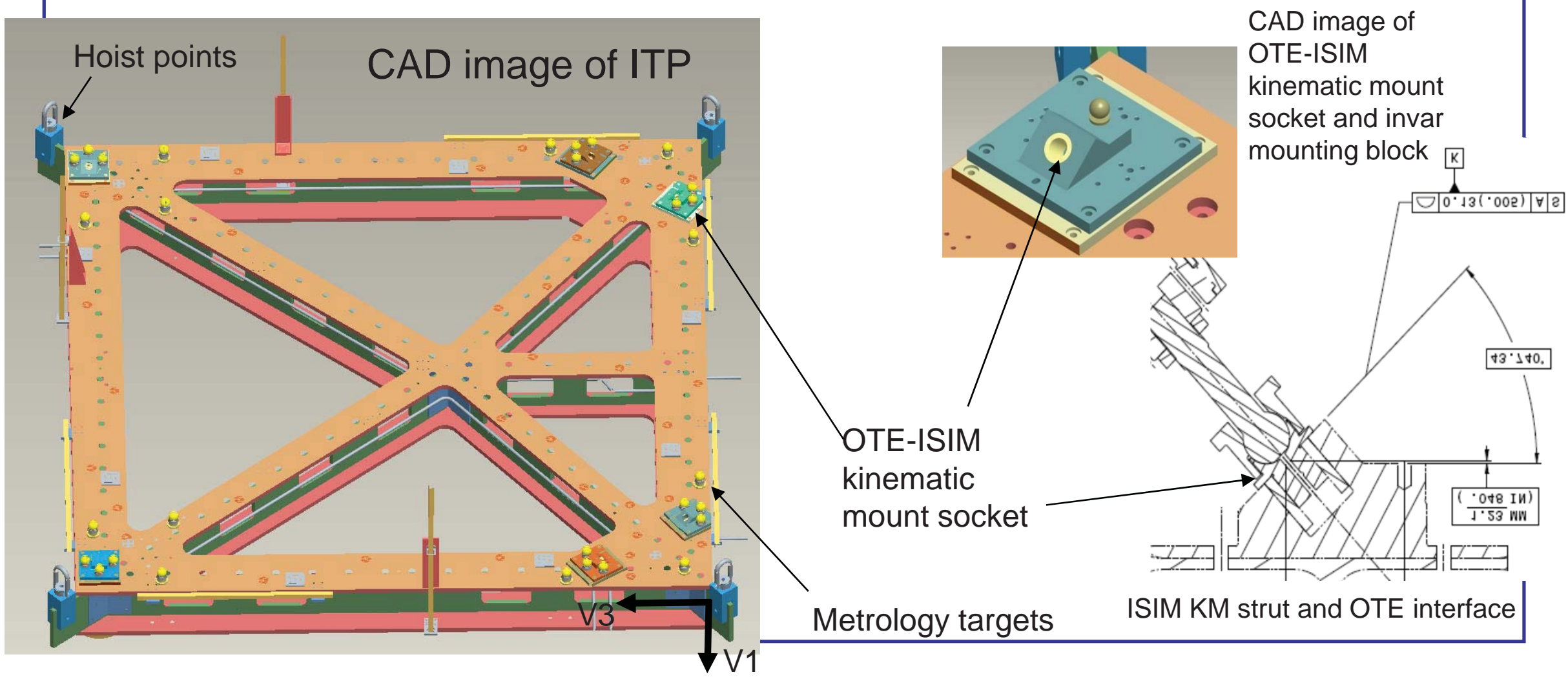




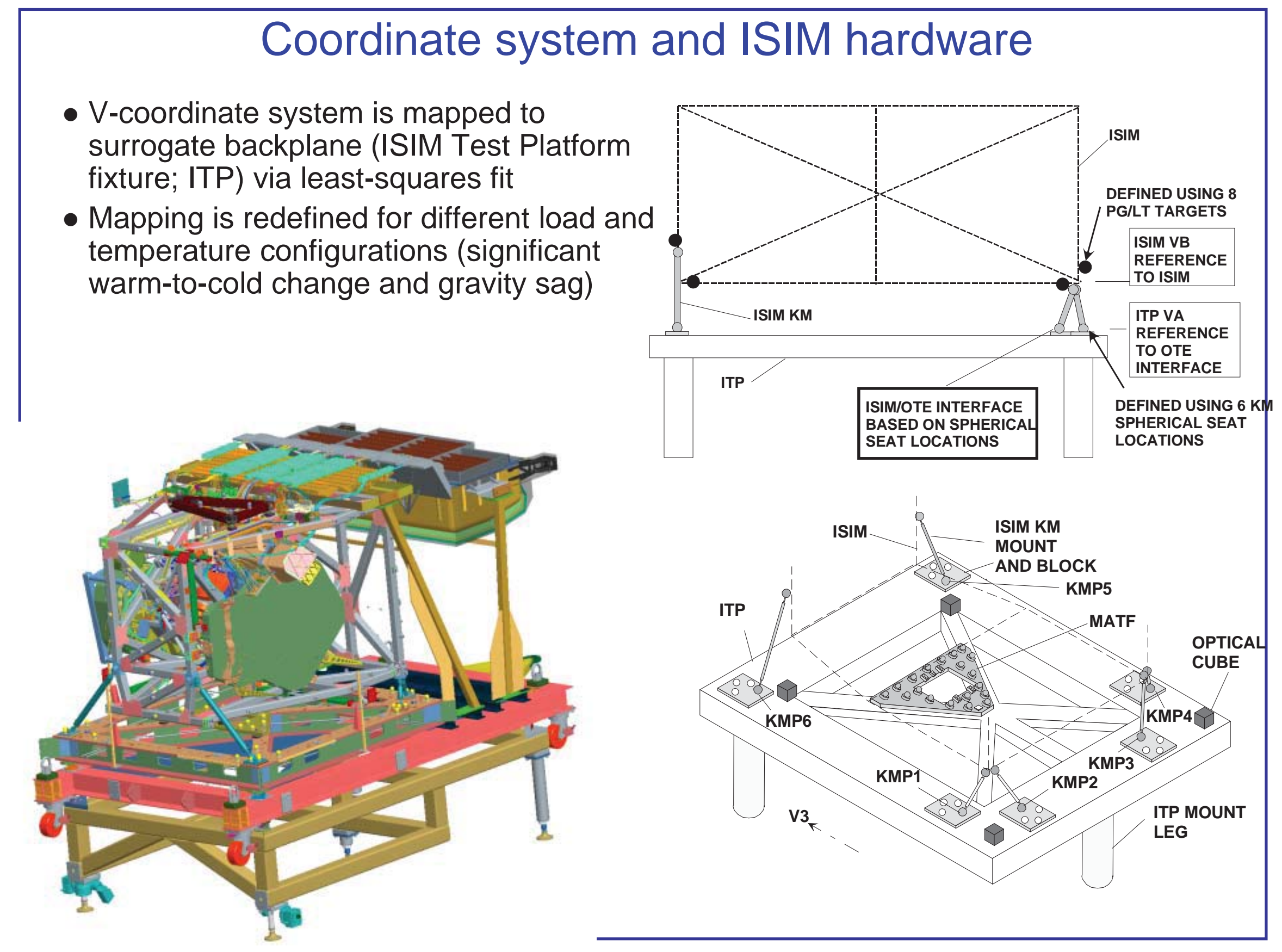




\section{ITP calibration, ambient}

- Ambient calibration of ITP

- Definition of V-coordinate system using interface references and MICD

- Calibration of ITP metrology references using LT, theodolites, PG

- Cube normals are aligned to approximately represent axes of $\mathrm{V}$-coordinate system

- Changes to ambient calibration

- Various load cases (empty, bare Structure, Integrated ISIM, OSIM's BIA)

- Repeatability with handling and mounting

- MATF installation

- Calibrate 6 DoF alignment with respect to Vcoordinates

- Repeatability of attachment

○

ISIM Error Budget Report (JWST-RPT-008175) documents allocations for ITP metrology uncertainty (knowledge) and impact to flight hardware alignment
CAD image of ITP+ISSD+IMIS showing metrology references

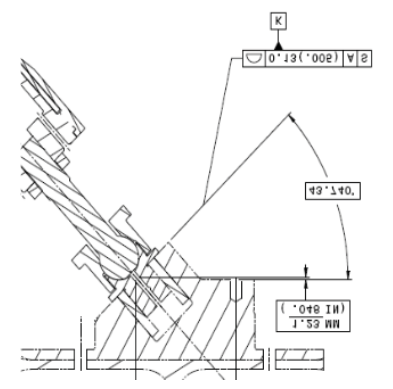

Extraction from MICD showing $1 \mathrm{KM}$ interface
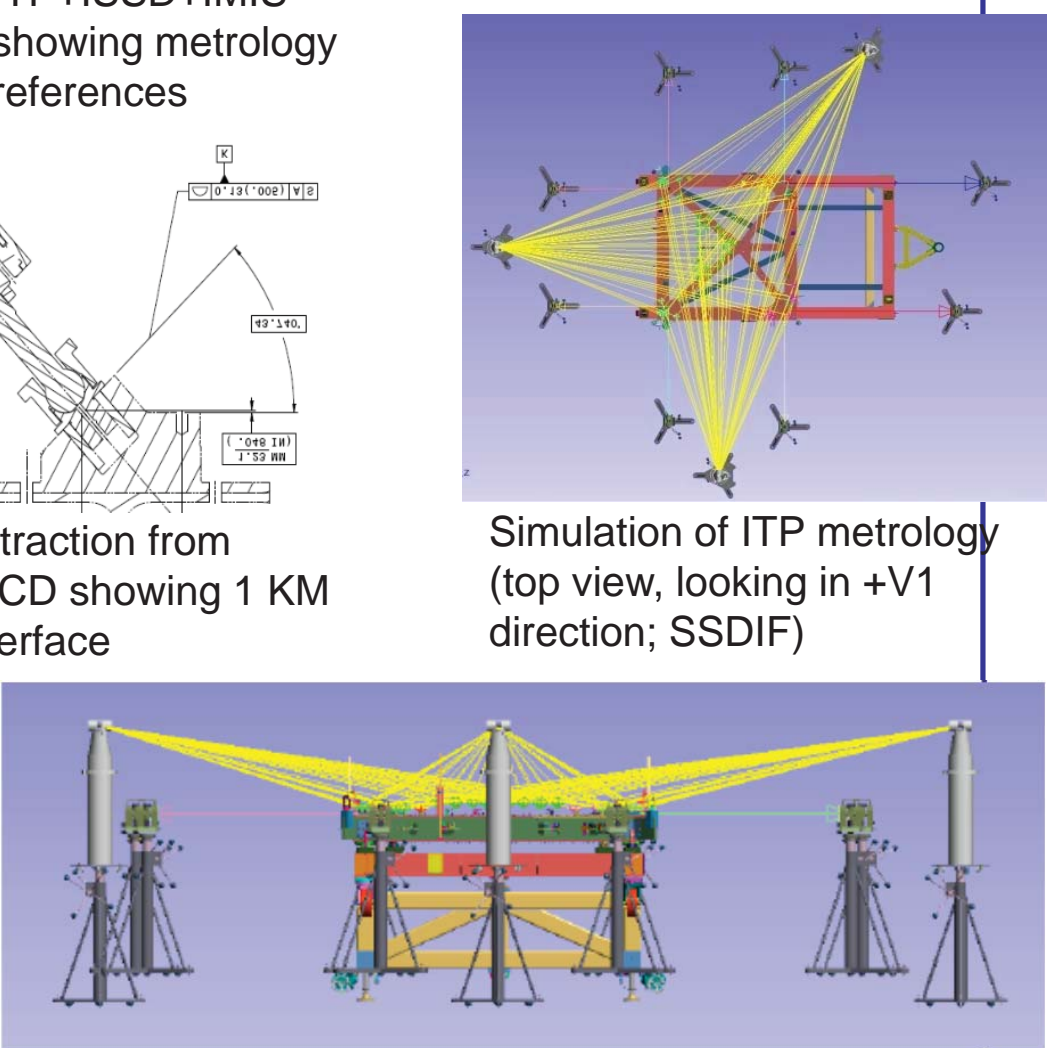

Simulation of ITP metrology (side view; SSDIF) 
Alignment of SI optical train relative to SI-ISIM interface is verified by SI developer using optomechanical tooling (i.e., ASMIF)

-

Levy requirements on Structure to avoid iterative compensated cryogenic alignment (i.e., no "windage" within Structure --- no pre-alignment at ambient to achieve correct placement at cryogenic operating temperature)

- Place SI-Structure interface plats on the ground at ambient at their nominal on-orbit alignment positions and orientations --- differences between warm vs. cold structure and loaded vs. Og structure are small and captured in error budget

- Measure bare Structure cryogenic performance to verify that it meets alignment requirements and increase confidence in Integrated ISIM performance (Structure's "cryo-set" test)

\section{Alignment approach for SI-to-Structure}

(Ambient Science Instrument Mechanical Interface Fixture; ASMIF)

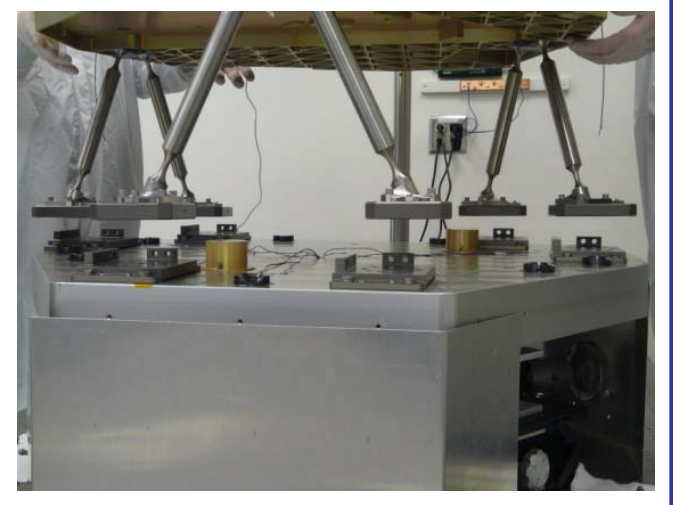

FGS ETU with ASMIF at COM DEV, Ottawa

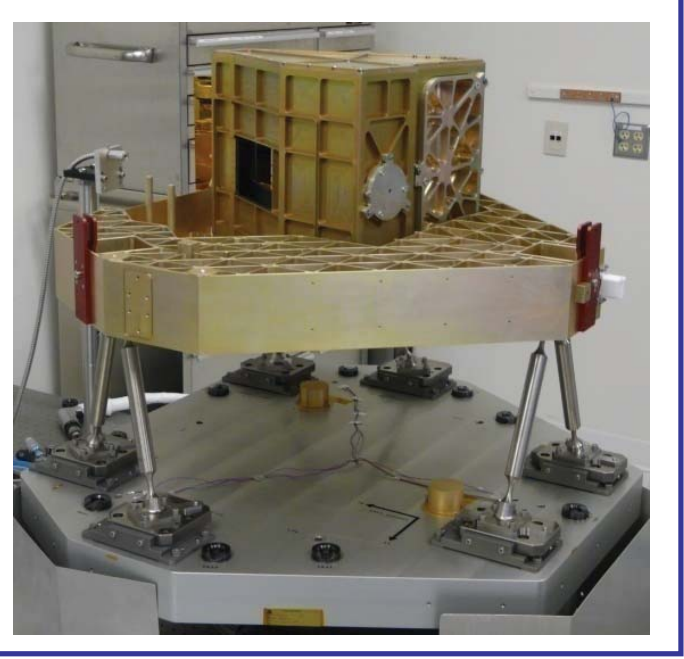




\section{Ambient integration of SIs with Structure}

- SIs are integrated to Structure

- Integrated ambient baseline metrology performed after Structure is populated with SIs: SI optical bench and ISIM Structure targets are measured using laser trackers and theodolites at ambient temperature under 1-g

- Measurements are compared with expectations based on

- SI+ASMIF metrology results

- As-built ITP, Structure, and SI validated mechanical models (e.g., gravity sag, ITP distortion)

- Measurements, including uncertainty, must agree with as-built mechanical models and "blueprints"

- This step ensures that SIs are where they should be in Structure at ambient under 1-g

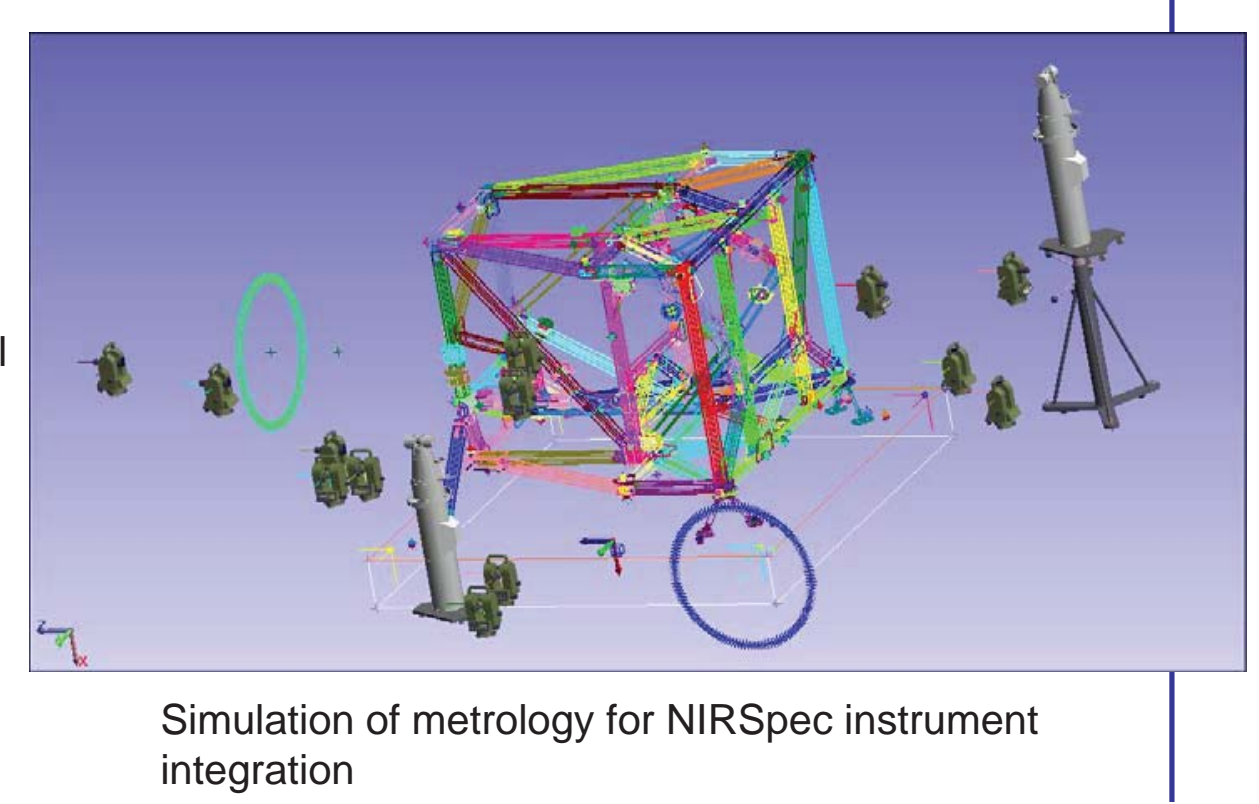

CAD view of Integrated ISIM showing NIRSpec side of assembly

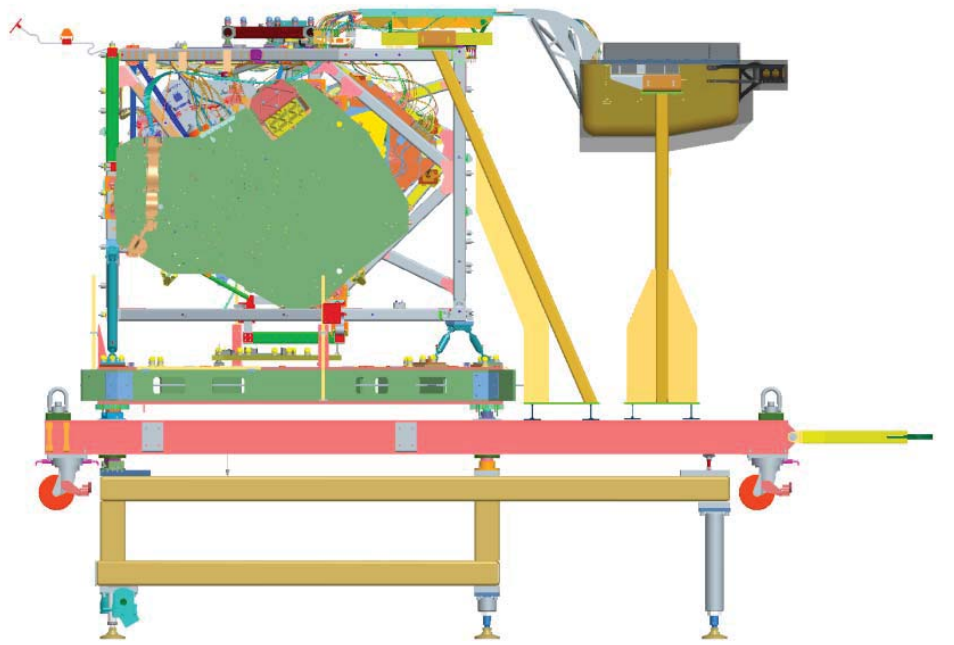

